\title{
The Invasive Species Commelina benghalensis L.: A Step Towards The Biological Flora of Egypt
}

\author{
Hoda A. Abd El-Hamid ${ }^{1}$, Mona M. El Bous ${ }^{2 *}$ \\ ${ }^{1}$ Botany Department, Faculty of Science Suez Canal University, Ismailia, Egypt \\ ${ }^{2}$ Botany Department, Faculty of Science Port-Said University, Port-Said, Egypt
}

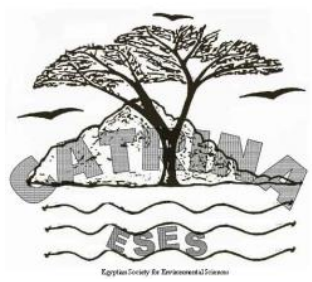

Invasive species Commelina benghalensis L. was investigated for its morphological, anatomical aspects. Ecological studies were carried out to explore the floristic composition of its community type and assess the factors that affect its invasion to the study area. Furthermore, proximate analysis, mineral composition, phytochemical screening and GC/MS analysis of secondary metabolites were studied to explore the nature of compounds present and evaluate its potential uses. Forty-seven weed species related to 21 families were recorded in $C$. benghalensis community type. Poaceae, Asteraceae and Brassicaceae were the most important families. Portulaca oleracea, Cyperus rotundus, Dactyloctenium aegyptium and Euphorbia heterophylla were the common associates. Therophytes were the most frequent life-form $(78.7 \%)$. The chorological analysis showed the prevalence of paleotropical, cosmopolitan and Mediterranean taxa. A canonical correspondence analysis (CCA) indicated that $\mathrm{pH}$, cations (calcium, potassium, sodium and magnesium), anions (bicarbonates, sulphates and chlorides), soil texture (sand, silt and clay) and organic matter were the most effective soil variables for the distribution of C. benghalensis and its associated species in the study area. The highest values of succulence and mean leaf surface area were recorded in mango orchards, while the highest shoot length and phytomass were registered in the habitats of crop fields. The results also indicated that this plant is a valuable source of nutritional, mineral and phytochemical compounds; hence it can be used as potential source of relatively low-cost, palatable forage for livestock and might be of a very important medicinal value and should be explored further to extract new drugs. It should not be included in the harmful weeds.

Keywords: Invasive species, plant communities, secondary metabolites, proximate analysis, chorology.

\section{INTRODUCTION}

Commelina benghalensis L. is an aggressive invasive weed (Wilson, 1981; Burns, 2004 and Heneidy, 2010) that produces both aerial and subterranean cleistogamous flowers (Isaac et al., 2013). Its serious growth habit allows to form dense pure stands, they may compete easily with low growing crops such as vegetables, cereals and legumes by smothering them (Holm et al., 1977). Therefore, it is a highly competitive and difficult to control weed (Ferrell et al., 2006).

Commelinaceae is the fifth largest family of Monocotyledons in Tropical Africa (Faden and Hunt, 1991). It is a family of 40 genera and over 600 species distributed from Northern temperate to tropical climates (Webster et al., 2005). This family is separating among families that truly debilitate crop performance (Daehler, 1998). Genus Commelina belongs to the Tribe Commelineae of subfamily Commelinoideae (Reveal and Chase, 2011).

Commelina species are ranked among the worst and extensively disseminated weed species. It is emerging as a potential threat for cultivated land (Isaac et al., 2013), those intensive spread of Commelina spp. are attributed to the viable seeds being produced both above and below ground. They also possess the ability to root at the nodes and can be propagated from cut stems. Genus Commelina is considered as one of the most important troublesome weed in 25 different crops in 28 countries (Webster et al., 2005). In addition, it boosts economic losses and environmental defect (Rocha et al., 2007).

In Egypt, family Commelinaceae is represented by 3 genera and 6 species (Boulos, 2005). Commelina benghalensis was originally known in Egypt as a very rare species recorded in Gebel Elba (Täckholm, 1974), subsequently it had been reported to be widely distributed, spread rapidly as weed in crop fields of Egypt (Boulos, 2005). Abd El-Ghani and Abdel-Khalik (2006) recorded this plant from Wadi Aideib and Wadi Yahameib of Gebel Elba Natural Reserve. It has been also recorded by Shaltout (2014) among the alien weeds in the Egyptian flora. In addition; It was reported by Abd ElGawad (2014) among invasive plants in some newly reclaimed areas in Egypt.

Weeds compete with cultivated crops and forages for moisture, light, and nutrients, but many weeds are nutrient-rich, digestible and of medicinal benefits (Lewis and Green, 1995). The composition of arable weed species is ruled by environmental conditions such as temperature and precipitation. Climatic change may become one of the most important determinants for the distribution of arable weeds (Peters et al., 2014). Weed communities are affected by soil characteristics (Pinke et al., 2010), allelopathic interactions (Mucina, 1997), agricultural practices and crop type (Andersson and Milberg, 1998 and Andreasen and Skovgaard, 2009).

Commelina benghalensis has been reported in folkloric medicine to poses antimicrobial, anti-inflammatory, analgesic and abortifacient properties (Raquibul Hasan et al. 2010; Cuéllar Cuéllar and Okori, 2010). Several studies have been held to investigate its phytochemical constitutes. Del Pero Martínez and Swain (1985) confirmed that flavone $\mathrm{C}$-glycosides are the dominant compounds in Commelinaceae. However, Chioma and 
Omoregie (2010) reported that phytochemical screening of $C$. benghalensis revealed the presence of phlobatannins, carbohydrates, tannins, glycosides, volatile oils, resins, balsams, flavonoids and saponins.

Mbazima (2009) assessed the effect of crude Methanolic extract of Commelina benghalensis on cancer cell growth to validate the traditional use of C. benghalensis as an anticancer agent; in the same context, Mokgotho (2009) proved antiproliferative activity of both the n-hexane and DCM extracts of $C$. benghalensis against Wil-2 NS cancerous cells. Raquibul Hasan et al. (2010) evauated the Analgesic potential of Commelina benghalensis and thereby justified its traditional uses in various types of pain. In addition the potential use of the species as a feed supplement for ruminants was proposed by Lanyasunya et al. (2008).

It was noticed that livestock in Ismailia governorate feed greedily on Commelina benghalensis; this observation formed the basis for the present study which aimed at 1) exploring the morphological, anatomical, taxonomical and phytochemical characteristics of Commelina benghalensis in order to redirect focus on the usefulness of the plant; 2) Studying the floristic composition and evaluating the environmental factors controlling its distribution; 3 ) determining the possible bioactive compounds and evaluate its nutritional value.

\section{MATERIALS AND METHODS}

\section{Study Area}

The study area located in Ismailia Governorate, between longitudes $31^{\circ} 40^{\prime}-32^{\circ} 38^{\prime} \mathrm{E}$ and latitudes $30^{\circ}$ $15-30^{\circ} 57^{\prime} \mathrm{N}$ (Map 1). It belongs to the Eastern Nile Delta that has a number of geomorphological features directly affecting the agricultural activities and land use. The soil of the study area is related to river terraces of fluvial and deltaic origins and wind-blown deposits according to FAO (1964) and Younes et al. (1977). The study area belongs to the arid province (Ayyad and Ghabbour, 1986). The mean monthly air temperature varies between $13.03^{\circ} \mathrm{C}$ in January and $27.31^{\circ} \mathrm{C}$ in July. The relative humidity varies from $64.75 \%$ in January to $51.15 \%$ in April. The highest precipitation (26.04 mm) was recorded in November, while the lowest $(0.76 \mathrm{~mm})$ was recorded in July. The mean evaporation rate varies between $3.8 \mathrm{mms} /$ day and $9.8 \mathrm{mms} /$ day (Ibrahim, 2017).

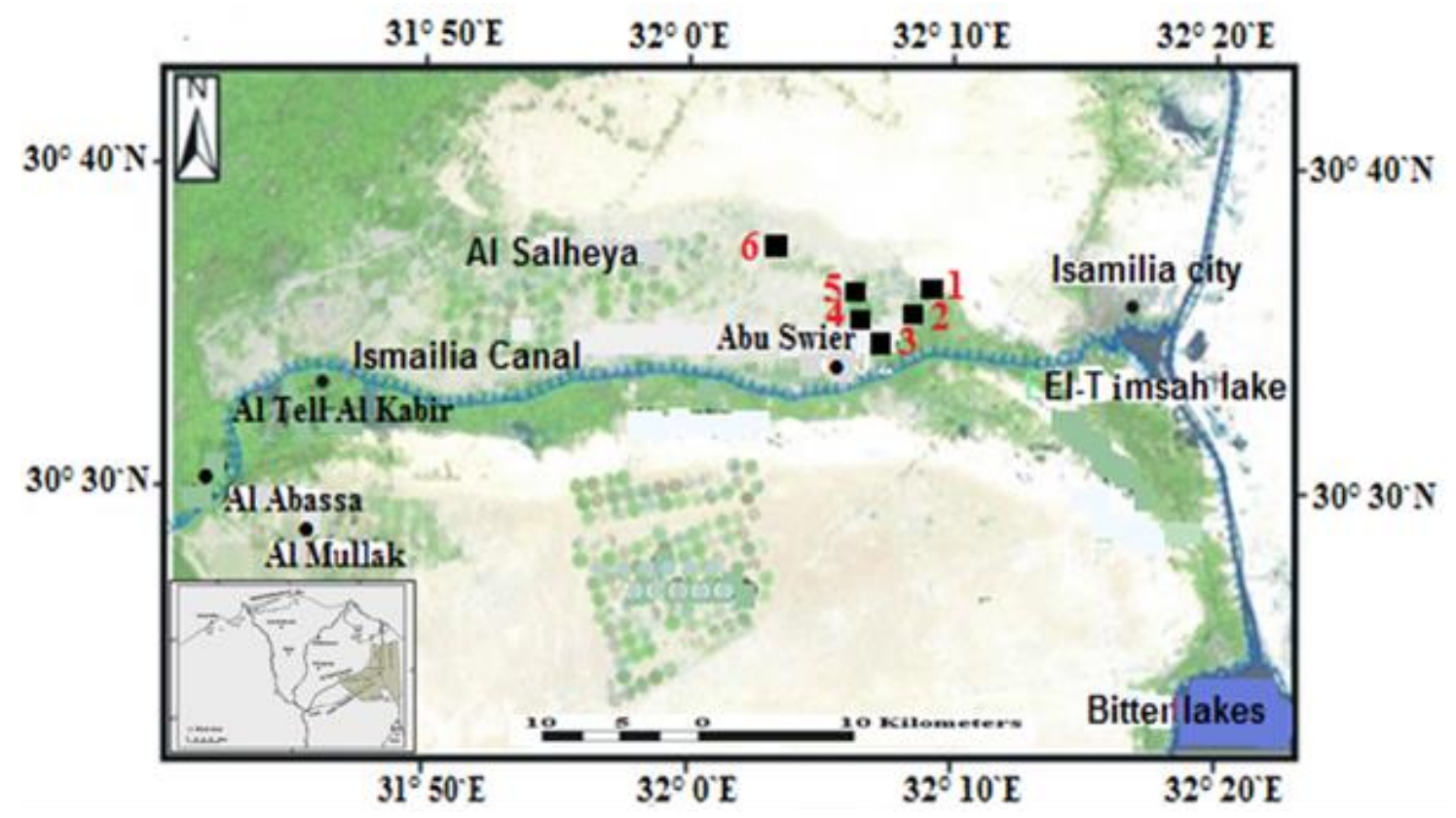

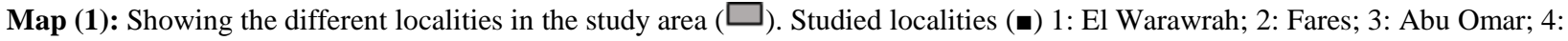
Abu Ragieh; 5: Abu Radwan; 6: Abu Kharwa.

\section{Morphology}

Fresh plant materials were collected from their natural habitats and deposited at the Herbarium of Suez Canal University (SCUI). To study the morphological characteristics of Commelina benghalensis, fresh and dried specimens were studied and described according to Boulos (2005). Olympus sz61 Stereomicroscope equipped with Optica View 7.3.1.7. En Camera was used for inspecting and collecting morphological data.

\section{Scanning Electron Microscopy (SEM)}

Freeze-fractured, and then freeze-dried. The specim- enswere sputter-coated with gold palladium and viewed with a JEOL JSMT-5200 scanning electron microscope operated at $25-30 \mathrm{Kv}$.
Anatomy
About $5 \mathrm{~mm} \times 5 \mathrm{~mm}$ portions were cut from fresh matured and well expanded leaves and stem, then immediately immersed in formalin-glacial acetic acid- ethanol solution (70\% FAA) for 24 hours. Thin cross- sections were made as described by (Johansen, 1940). Well stained sections were examined under light microscope and photographed using a Zeiss Camera to 
calculate the means and standard error among the different cross-sections. Different references book were used for anatomical description (e.g., Cutler et al., 2008).

\section{Vegetation analysis}

Twenty stands (area $=10 \mathrm{~m} \times 10 \mathrm{~m}$ each) dominated by Commelina benghalensis were surveyed at summer and autumn seasons of the year 2016. The stands covered three habitat types in six localities of the study area namely; newly cultivated mango orchards (MN), old cultivated mango orchards (MO) and crop fields (CF). The density was measured by counting the number of individuals of species within each stand and the cover was estimated by using the line-intercept method (Canfield, 1941). The relative values of density and cover of each species were calculated and summed up to give an estimate of its importance value (IV out of 200). The taxonomic nomenclature of the species in the study area was given according to Boulos (1999, 2002, 2005, 2009). Life form of each species was listed according to Raunkiaer (1934). The phytogeographical range of species distribution was carried out according to Good (1974), Wickens (1976) and Abd El-Ghani (1981 and 1985). The presence value (P) of each species was expressed as the number of stands in which a plant species is present in relation to the total number of sampled stands. Phytomass of the studied plant was determined as dry weight per unit area by harvesting plants in five quadrats $\left(0.5 \mathrm{~m}^{2}\right)$ according to Shukla and Chandel (1996). Fresh (FW) and dry (DW) weights were determined and expressed as $\mathrm{g} / \mathrm{m}^{2}$. The succulence was calculated as a ratio between fresh weight and dry weight (FW/DW) (Khedr and Hegazy, 1998). The average leaf area was measured according to the equation of Kemp (1960): $A=K \times L \times B$, where $K$ is Kemp's constant, it equals (0.9) for monocots, $\mathrm{L}$ and $\mathrm{B}$ is leaf length and leaf width at mid-point respectively.

\section{Soil analysis}

Three soil samples were collected from each stand at a depth of 0-50 cm, mixed, air dried and passed through a $2 \mathrm{~mm}$ sieve to separate gravel and debris. Soil texture was analyzed using the Bouyoucos hydrometer method (Bouyoucos, 1962). Organic matter content was estimated by loss on ignition method according to Allen et al. (1974). Determination of calcium carbonate content was carried out using Collin's Calcimeter (Allen et al., 1974). Soil salinity (EC) and soil reaction $(\mathrm{pH})$ were estimated in (1:5) soil-water extract using a digital conductivity meter (Model 76, ES and D, Inc. USA and a digital pH-meter (Model 201, Orion research, USA) respectively. Carbonates $\left(\mathrm{CO}_{3}^{--}\right)$and bicarbonates $\left(\mathrm{HCO}_{3}{ }^{-}\right)$were determined volumetrically (Pierce et al., 1958). Chlorides $\left(\mathrm{Cl}^{-}\right)$were estimated according to Baruah and Barthakur (1997). Sulphates were determined by the gravimetric method using barium chloride (Piper, 1942). Calcium and magnesium were estimated according to Baruah and Barthakur (1997). Estimation of sodium and potassium were carried out using a flame photometer (Model 410, Corning, England) as described by Allen et al. (1974).

\section{Data analysis}

The relationships between the vegetation and the soil gradients of the three studied habitats were assessed using Canonical Correspondence Analysis (CCA) (Ter Braak, 1986 and 1994). The input data in this analysis were in two forms: stands versus species importance values (IV) data matrix and stands versus soil variables data matrix. Statistical analysis was carried out using SPSS version 22.0 for windows software.

\section{Proximate composition}

Moisture, total ash content, crude protein, fat, total carbohydrates and crude fiber content were estimated according to the procedures described in AOAC (1990). Ether extract (crude fat) was determined by the Soxhlet extraction (Harborne, 1984).

\section{Mineral content}

The macro and micro-elements were estimated in the ash of the Commelina benghalensis. $\mathrm{Mg}, \mathrm{Fe}, \mathrm{Cu}, \mathrm{Mn}$ and $\mathrm{Zn}$ were measured using atomic absorption (PerkinElmer atomic absorption Spectrophotometer 2380). Phosphorus (P) was determined by applying molybdenum blue method using a spectrophotometer (T60 UV/VIS Spectrophotometer). Total nitrogen (N) was assessed by the Kjeldahl method. All these procedures are according to (Allen et al., 1974). The calculation of nutritive value (kcal/100 g dry weight) was carried out using the Atwater system as described by the World Health Organization (1985) by multiplying the values obtained for protein, carbohydrates and fat by 4.00, 3.75 and 9.00, respectively, and the results are expressed in k.cal.

\section{Phytochemical analysis}

Preliminary phytochemical screening of $C$. benghalensis extract was performed using standard procedures (Harborne, 1998).

\section{GC-MS analysis}

Gas Chromatography Mass Spectrometry (GC-MS) analysis separates all the components in the sample and provides a representative spectral output. The analysis was performed using GC Shimadzu QP2010 system and gas chromatograph interfaced to a Mass Spectrometer (GC-MS) equipped with Elite-1 fused silica capillary column. The GC-MS Conditions: Column: (Varian Chrompack CP-Sil 8, 30m length x 0.25mm ID). Carrier gas: Helium with constant flow, $1.0 \mathrm{ml} / \mathrm{min}$. Injector Temperature $=250^{\circ} \mathrm{C}$, Split Ratio $=2$. Oven Temperature: Program: Start at $40^{\circ} \mathrm{C}$ withhold time of $1 \mathrm{~min}$, then, 40 to $150^{\circ} \mathrm{C}$ at a rate of $10^{\circ} \mathrm{C} / \mathrm{min}$, with no hold, then, 150 to $280^{\circ} \mathrm{C}$ at a rate of $5^{\circ} \mathrm{C} / \mathrm{min}$ with a hold for 5 min. Total Runtime $=30 \mathrm{~min}$. Injected Volume of the extract $=1 \mu \mathrm{L}$. Interface Temperature $=280^{\circ} \mathrm{C}$.

The interpretation of the mass spectrum GC-MS was carried out using the database of National Institute Standard and Technology (NIST) having more than 62,000 patterns. The name, molecular weight and structure of 
the components of each sample were ascertained using NIST Ver. 2.1 MS data library. The spectrum of the unknown component was compared with the spectrum of the component stored in the NIST library version (NIST Chemistry Web Book) (Joulain and König, 1998) and a database of chemical molecules https://pubchem. ncbi.nlm.nih.gov/search/search.cgi.

\section{RESULTS}

\section{Morphological Characteristics}

Herbaceous, summer annual, 20-36 cm height. Stem slender, creeping to decumbent (Fig.1); fibrous rooting with subterranean cleistogamous flowers; internode 5$10 \mathrm{~cm}$; leaf-sheath $0.5-3 \mathrm{~cm}$ closed, ciliate at the mouth with purplish or white bristle along free edge with hooked or straight three cell non-glandular hairs; Leaves spirally arranged, $6-10 \times 2.5-5 \mathrm{~cm}$, ovate to ovate lanceolate, pubescent, acute, pseudo-petiole 0.4-1.8 cm; with 10-12 mm sheathy base with long hairs, leaf venation parallel convergent. Inflorescence leafopposed 1 cincinnus (scorpioid cyme); The cincinni enclosed in a folded spathe; spathes 1.5-2.2 $\times 1.8-3$ conduplicate, broader than length, fused thought their proximate margin ;sessile or shortly pedunculate; lower cincinnus enclosed within the spathe, with 2-3 bisexual flowers; cincinnus peduncle $0.8-1.2 \mathrm{~cm}$; pedicel $4-8$ $\mathrm{mm}$, protruding in flower, reflexed and enclosed within the spathe in fruit; upper cincinnus protruding from the spathe; peduncle $1.5-2 \mathrm{~cm}$, with 1 staminate flower; dimorphic flower, Chasmogamous (aerial) flowers, zygomorphic, borne in stalked, or rarely sessile pedicel 4-8 $\mathrm{mm}$; lower sepals $3.5 \times 2.5 \mathrm{~mm}$, oblong; upper sepal $3.5 \times 1.5 \mathrm{~mm}$, elliptic; lower petal blue; paired petals $0.7-0.85 \times 0.9-1.1 \mathrm{~cm}$, with $4-5 \mathrm{~mm}$ claw, blue; median stamen with filament $5-7 \mathrm{~mm}$; anther $2 \mathrm{~mm}$, lateral stamen with $5-7 \mathrm{~mm}$ filament; anthers $1.4 \mathrm{~mm}$, blue; staminodes with 4-4.5 mm filaments, antherodes yellow; stigma capitate blue. Capsule produced by chasmo- gamous flower 4.5-5.5× $3 \mathrm{~mm}$ oblongelliptic; Capsules produced by cleistogamous flowers (Fig. 2b) ellipsoid 5-7x3-4 mm splits along two valves (Fig. 2e), 2- seeded, pyriform, 3-5 $\mathrm{ml}$ at the soil surface, dehis-cent, each containing 1 or 2 seeds with circular hilum. Seeds dimorphic 3-5 per capsule with varying size; Aerial seeds 2.5-5 $\times 1.5-2.5 \mathrm{~mm}$ oblong, obtuse, brown, rugose, irregularly reticulate and dorsal longitudinal ridge, (Fig. 2a), hilum narrow; cleistogamous seeds (Fig. 2c-d) bigger 3.5-5.2× 1.2-2. Curved elliptic, nearly smooth, brown; ventral concave side with a central raised circular ridge (hilum), and a short shallow groove extending transversely. Fl. Jul.-Aug. and Fr. Aug.-Oct.

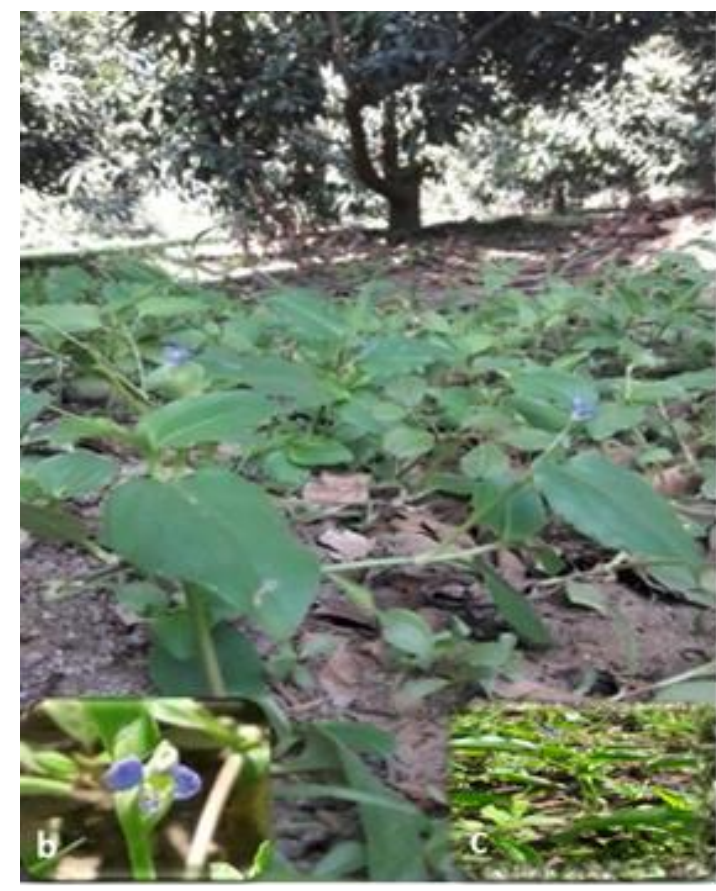

Figure (1): Commelina benghalensis (a) Close up view of Commelina benghalensis showing its habit, (b) flower, and (c) intensive plant growth.
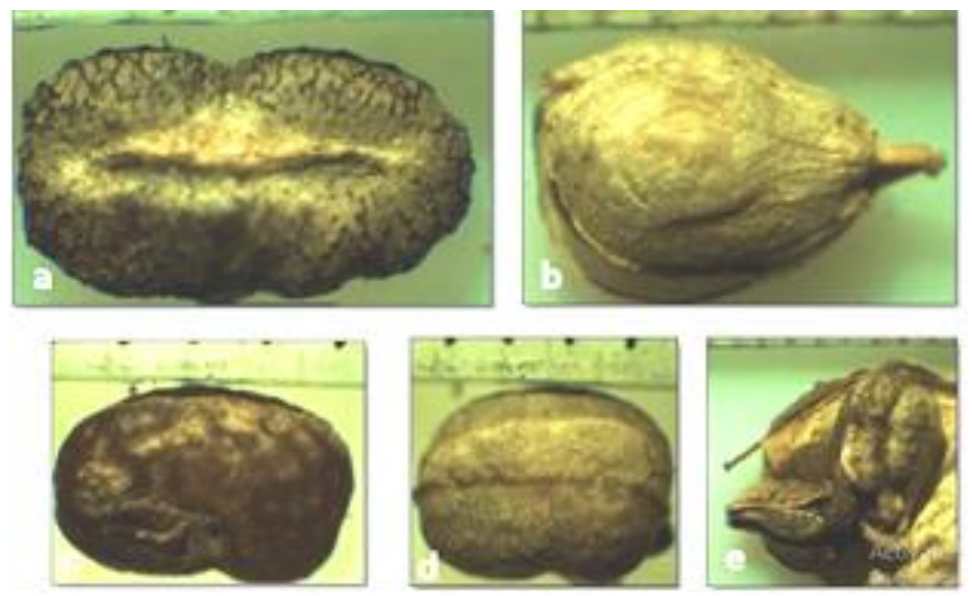

Figure (2): Fruiting parts of Commelina benghalensis. (a) Aerial seed showing irregular reticulate surface and longitudinal ridge, (b) Cleistogamous pyriform capsule, (c) Lateral view of cleistogamous seed, (d) Dorsal view showing concave side, and (e) Dehiscent capsule by valves. 


\section{Anatomical properties}

- Stem anatomy

The transverse section of stem has a primary structure (Fig. 3 b-d). The epidermis consists of a single layer of epidermal cells $(2.59 \pm 1.95 \mu \mathrm{m}$ diameter $)$ and contains paracytic stomata (Fig. 3c) covered with a thin cuticle at the same level of epidermal cells. Below the epidermis there is an angular collenchyma made up of 3-4 layers of cells, interrupted at the level of the stomata with the chlorenchyma. Cortex consists of circular and polygonal parenchyma ( $65.83 \pm 6.7 \mu \mathrm{m}$ diameter); below the cortex, one fiber ring is incorporated outer collateral vascular bundles. Exterior vascular bundles consist of phloem, metaxylem and protoxylem and surrounded by bundle sheath. The remaining vascular bundles are irregular arranged in the pith. The medullar vascular bundles are reduced to phloem and large Lysigenous lacuna $(61.87 \pm 2.6 \mu \mathrm{m}$ diameter) (Fig. 3d). The fibers ring has joined collateral vascular bundles located toward the cortex and others located toward the pith.

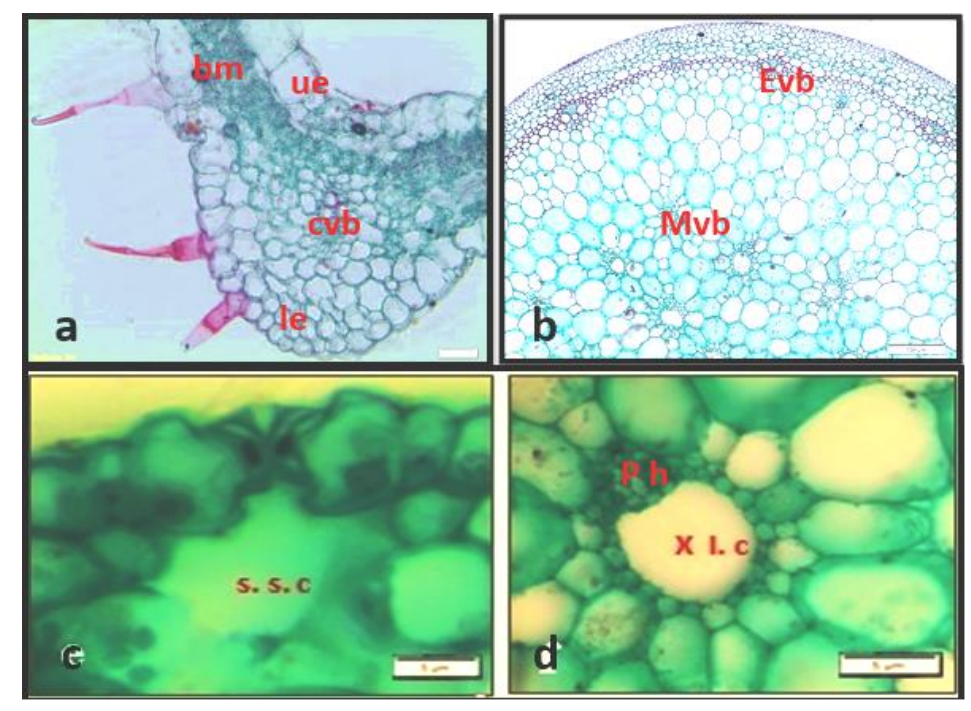

Figure (3): Anatomy of Commelina benghalensis L. (a) Leaf cross section (Scale bar $=50 \mu \mathrm{m})$. (b) Whole cross section of stem $($ Scale bar $=200 \mu \mathrm{m})$. (c) Enlarged part of stem epidermis showing stomata and sub-epidermal chamber. (d) Enlarged medullary vascular bundle. Abbreviations: C.V.B (central vascular bundle; b.m (bifacial mesophyl); U.E (upper epidermis); L.E (lower epidermis); C.V.B (central vascular bundle); E.V.B (external vascular bundle); M.V.B (medullary vascular bundle); S.S.C (substomatal chamber); PH (phloem); X.L.C (xylem lysigenous cavity).

\section{- Leaf anatomy}

Lamina thickness $356.7 \pm 2.79 \mu \mathrm{m}( \pm$ : standard error). The epidermis $(69.05 \pm 2.85)$ usually constituting less than $20 \%$ of the leaf thickness, macrohairs present on the abaxial surface include eglandular uniseriate multicellular hairs with tapering or hook shaped distal cell; papillae is restricted to the leaf margin (Fig. 3a-d). Epidermal cells of both surfaces are arranged in a single-layer of relatively large epidermal cells $(69.05 \pm$ $2.85 \mu \mathrm{m}$ ) in length, $78.45 \pm 5.29 \mu \mathrm{m}$ in width, with thin walls rectangular and elongated perpendicularly to the surface. Stomata on the lamina are restricted to the abaxial surface and located at the same level of epidermal cells with substomatal chamber. Bifacial Mesophyll (Fig. 3a) is differentiated into one row of palisade $(41.89 \pm 5.07 \mu \mathrm{m})$ with abundant chloroplast toward the adaxial surface and 3-4 row of isodiametric and irregular shape spongy parenchyma $(35.34 \pm 1.84$ $\mu \mathrm{m})$ with abundant chloroplast and wide intercellular spaces. Relatively macro-uniseriate hair (182.41 \pm $16.389 \mu \mathrm{m})$ with pointed distal cells and hooked hair (Fig. 4a-c) are present on abaxial surface and tubular leaf sheath. Papillae and trichomes are restricted to the leaf margin (Fig. 4b). Leaves are dorsiventral, hypostomatic, with hexacytic stomatal complex (Fig. 4d). Lamina is crossed by a parallel venation. The collateral vascular bundles are distributed in the middle region of mesophyll, surrounded by the pericycle and the endodermis.

\section{Ecological results \\ Floristic composition}

The recorded species in the Commelina benghalensis community type were 47 species belonging to 41 genera and related to 21 families. Poaceae, Asteraceae, Brassicaceae and Euphorbiaceae were the most common families as they contribute more than $49 \%$ of the recorded species (Table 1). The recorded species were classified into 42 annuals (89.4\%), 4 perennials (8.5\%) and one biennial species. Based on the life-forms, these species were grouped under three types: therophytes (37 species $=78.7 \%)$, hemicryptophytes $(8$ species $=17.1 \%)$ and geophytes $(2$ species $=4.3 \%)$. Paleotropical taxa were the main chorotype (13 species) followed by Cosmopolitan and Mediterranean taxa (12 species each). Pantropical and Neotropical taxa represented by 8 and 2 species, respectively. 

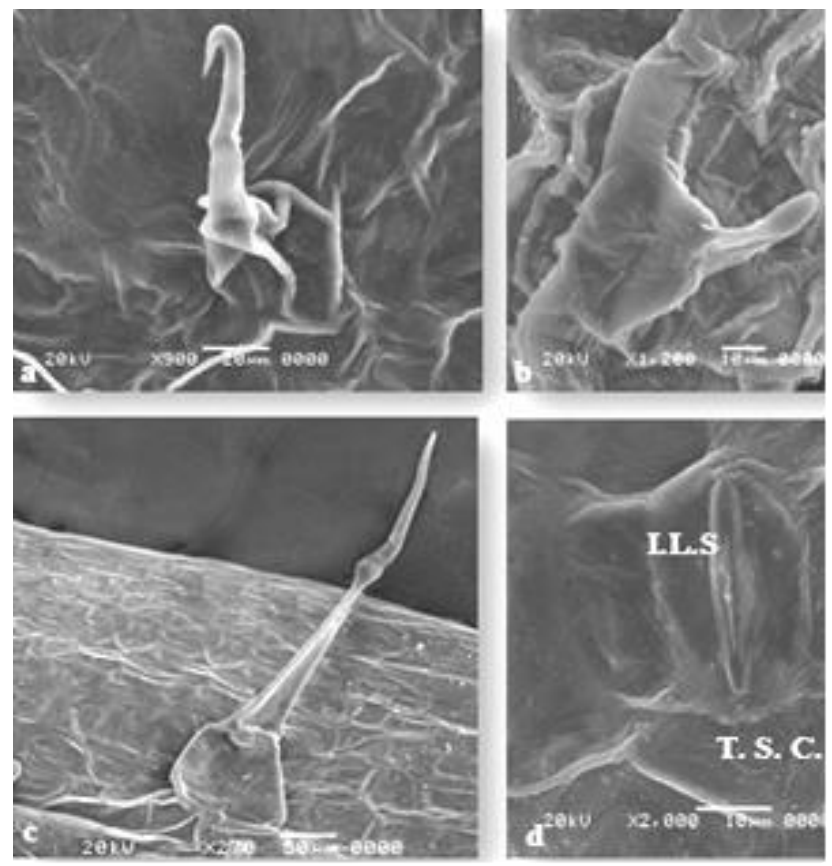

Figure (4): SEM photograph of abaxial surface of Commelina benghalensis leaf. (a) Hooked hair. (b) one-celled papillae on the leaf margin. (c) Eglandular unisriate multicellular hair with pointed distal cell. (d) enlarged view of paracytic stomata showing terminal subsidiary cell (T.S.C.); innermost lateral subsidiary cell (1. S. C.); outermost lateral subsidiary cell (O.L.S).

\section{Vegetation analysis}

Table (2) shows the mean importance values of Commelina benghalensis and its associated species in the three studied habitats. Notably, Commelina benghalensis was the dominant species in the three habitats. It attained the highest importance values $(65.8,47.2$ and 98.79 ) in old cultivated mango orchards (MO), newly cultivated mango orchards (MN) and crop fields (CF) habitat), respectively. The most common associated species in old cultivated mango orchards habitat (MO) were Galinsoga parviflora $(\mathrm{IV}=37.1)$ and Digitaria sanguinalis (IV=11.3). While, Euphorbia heterophylla (IV=37.4), Ipomea hederacea (IV=32.1), Cynodon dactylon (IV=20.7) and Bidens pilosa (IV=10.1) represented the most common associated species in newly cultivated mango orchards habitat (MN). The most common associated species in crop fields habitat (CF) includes Portulaca oleracea ( $\mathrm{IV}=18.5)$, Cyperus rotundus $(\mathrm{IV}=13.6)$ and Dactyloctenium aegyptium $(\mathrm{IV}=10.4)$.

\section{Soil characteristics}

The soil characteristics of the habitats supporting Commelina benghalensis community type are shown in table (3). Results show that stands of old cultivated mango orchards (MO) characterized by the highest levels of sand fractions $(79.09 \%)$, organic matter $(1.40 \%)$, electrical conductivity $(0.84 \mathrm{~ms} / \mathrm{cm})$, chlorides $(56.81$ ppm) and magnesium $\left(24.71 \mathrm{mg} 100 \mathrm{~g}^{-1}\right)$, but the lowest values of silt $(7.87 \%)$, clay $(13.04 \%)$, water holding capacity $(45.80 \%)$, calcium carbonates $(1.45 \%), \mathrm{pH}$ (8.39) and bicarbonates (94.69 ppm). Newly cultivated mango orchards stands (MN) had the highest values of clay $(16.27 \%)$, water holding capacity $(54.00 \%), \mathrm{pH}$ (8.55), calcium carbonates $(1.94 \%)$, sulphates (648.33 ppm) and calcium cation (626.67 mg $100 \mathrm{~g}^{-1}$ ) but the lowest values of sand $(74.40 \%)$, organic matter $(0.79$ $\%)$, electrical conductivity $(0.65 \mathrm{~ms} / \mathrm{cm})$, chlorides (47.37 ppm), magnesium (24.00 mg $100 \mathrm{~g}^{-1}$ ), sodium $\left(3.60 \mathrm{mg} 100 \mathrm{~g}^{-1}\right.$ ) and potassium $\left(8.47 \mathrm{mg} 100 \mathrm{~g}^{-1}\right)$. Soil of crop fields habitat $(\mathrm{CF})$ had the highest values of silt $(9.59 \%)$, soluble carbonates (1.71 ppm), bicarbonates (105.24 ppm), sodium (4.43 mg100 g $\mathrm{g}^{-1}$ ) and potassium (9.46 mg100 $\left.\mathrm{g}^{-1}\right)$, but the lowest of sulphates (172.83

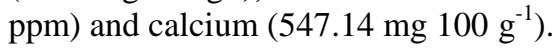

The relationships between Commelina benghalensis and its common associated species with the soil variables are shown on the ordination diagram produced by Canonical Correspondence Analysis (CCA) of the biplot of species-soil variables (Fig. 5). It is obvious that, $\mathrm{pH}$, cations (calcium, potassium, sodium and magnesium), anions (bicarbonates, sulphates and chlorides), soil texture (sand, silt and clay) and organic matter were the most effective soil variables on the distribution of $\mathrm{C}$. benghalensis and its common associated species in the different habitats. Cyperus rotundus and Portulaca oleracea showed strong relationship with silt fraction, $\mathrm{CaCo} 3$, sodium, soluble carbonates and potassium. While, C. benghalensis and Dactyloctenium aegyptium exhibited a clear relationship with bicarbonates, organic matter, electrical conductivity and sand fraction. The sulphates, water holding capacity, clay fraction, calcium and magnesium are the most effective variables on Euphorbia heterophylla and Digitaria sanguinalis. On the other hand, Bidens pilosa, Ipomea hederacea and Galinsoga parviflora are only affected by chloride. 
Abd El-Hamid H. A. \& El Bous M. M.

Table (1): Floristic composition of the Commelina benghalensis community type

\begin{tabular}{|c|c|c|c|c|}
\hline Species & Life span & Life form & Floristic category & $\mathbf{P}(\%)$ \\
\hline Aizoaceae & & & & \\
\hline $\begin{array}{l}\text { Trianthema portulacastrum } \mathrm{L} . \\
\text { Amaranthaceae }\end{array}$ & Ann & $\mathrm{H}$ & PAN & 30 \\
\hline Amaranthus hybridus L. & Ann & Th & PAL & 45 \\
\hline Amaranthus lividus L. & Ann & $\mathrm{Th}$ & $\mathrm{ME}+\mathrm{IR}-\mathrm{TR}$ & 15 \\
\hline Chenopodium album $\mathrm{L}$. & Ann & Th & COSM & 5 \\
\hline $\begin{array}{l}\text { Chenopodum murale } \mathrm{L} . \\
\text { Asclepiadaceae }\end{array}$ & Ann & Th & COSM & 35 \\
\hline $\begin{array}{l}\text { Cynanchum acutum } \mathrm{L} \text {. } \\
\text { Asteraceae }\end{array}$ & Per & $\mathrm{H}$ & $\mathrm{ME}+\mathrm{IR}-\mathrm{TR}$ & 10 \\
\hline Bidens pilosa $\mathrm{L}$. & Ann & Th & PAN & 45 \\
\hline Conyza bonariensis (L.) Cronquist & Ann & Th & NEO & 5 \\
\hline Eclipta prostrata (L.) L. & Ann & Th & NEO & 5 \\
\hline Galinsoga parviflora Cav. & Ann & Th & COSM & 45 \\
\hline Sonchus oleraceus L. & Ann & Th & COSM & 30 \\
\hline $\begin{array}{l}\text { Xanthium strumarium L. } \\
\text { Brassicaceae }\end{array}$ & Ann & Th & COSM & 5 \\
\hline Brassica tournefortii Gouan. & Ann & Th & $\mathrm{ME}+\mathrm{IR}-\mathrm{TR}+\mathrm{SA}-\mathrm{SI}$ & 5 \\
\hline Capsella bursa-pastoris (L.)Medik & Ann & Th & COSM & 5 \\
\hline Cronopus didymus (L.) Sm & Ann & Th & COSM & 10 \\
\hline $\begin{array}{l}\text { Erucastrum arabicum Fisch.\&C.A. Mey. } \\
\text { Caryophylaceae }\end{array}$ & Ann & Th & SA-SI+S-Z+PAL & 20 \\
\hline $\begin{array}{l}\text { Stellaria pallida (Dumort.) Murb. } \\
\text { Cleomaceae }\end{array}$ & Ann & Th & ME+ER-SR & 10 \\
\hline $\begin{array}{l}\text { Gynandropsis gynandra (L.) Briq } \\
\text { Commelinaceae }\end{array}$ & Ann & $\mathrm{Th}$ & PAL & 45 \\
\hline $\begin{array}{l}\text { Commelina benghalensis } \mathrm{L} . \\
\text { Convolvulaceae }\end{array}$ & Ann & $\mathrm{H}$ & PAL+SA-SI & 100 \\
\hline Convolvulus arvensis L. & Per & $\mathrm{H}$ & COSM & 15 \\
\hline Ipomoea hederacea Jacq. & Ann & $\mathrm{H}$ & $\mathrm{PAL}+\mathrm{NEO}$ & 15 \\
\hline $\begin{array}{l}\text { Ipomoea obscura (L.) Ker Gawl. } \\
\text { Cyperaceae }\end{array}$ & Ann & $\mathrm{H}$ & PAL+ SA-SI & 5 \\
\hline $\begin{array}{l}\text { Cyperus rotundus L. } \\
\text { Euphorbiaceae }\end{array}$ & Per & G & PAN & 55 \\
\hline Euphorbia helioscopia L. & Ann & Th & $\mathrm{ME}+\mathrm{IR}-\mathrm{TR}+\mathrm{SA}-\mathrm{SI}$ & 5 \\
\hline Euphorbia heterophylla $\mathrm{L}$. & Ann & $\mathrm{Th}$ & PAN & 60 \\
\hline Euphorbia hirta L. & Ann & $\mathrm{Th}$ & PAN & 5 \\
\hline $\begin{array}{l}\text { Euphorbia peplus } \mathrm{L} . \\
\text { Lamiaceae }\end{array}$ & Ann & Th & $\mathrm{ME}+\mathrm{IR}-\mathrm{TR}+\mathrm{ER}-\mathrm{SR}$ & 35 \\
\hline $\begin{array}{l}\text { Lamium amplexicaule } \mathrm{L} . \\
\text { Malvaceae }\end{array}$ & Ann & $\mathrm{Th}$ & ME+IR-TR+ER-SR & 10 \\
\hline Malva parviflora $\mathrm{L}$. & Ann & Th & ME+IR-TR & 20 \\
\hline $\begin{array}{l}\text { Sida alba } \mathrm{L} \text {. } \\
\text { Poaceae }\end{array}$ & $\mathrm{Bi}$ & Th & PAN & 10 \\
\hline Brachiaria deflexa (Schumach.) Robyns & Ann & Th & PAL & 10 \\
\hline Bromus catherticus Vahl & Ann & Th & COSM & 5 \\
\hline Cenchrus biflorus Roxb. & Ann & $\mathrm{Th}$ & NEO & 20 \\
\hline Cynodon dactylon (L.) Pers & Per & $\mathrm{G}$ & PAN & 15 \\
\hline Dactyloctenium aegyptium (L.)Willd. & Ann & Th & PAL & 50 \\
\hline Digitaria sangunalis (L.) Scop. & Ann & Th & PAL & 60 \\
\hline Echinocloa colona $(\mathrm{L}$.$) Link$ & Ann & Th & PAN & 25 \\
\hline Eluesine indica (L.) Gaerth. & Ann & Th & PAL & 20 \\
\hline $\begin{array}{l}\text { Setaria verticilata (L.) P. Beauv. } \\
\text { Polygonaceae }\end{array}$ & Ann & Th & PAL & 40 \\
\hline Emex spinosa (L.) Campd. & Ann & Th & $\mathrm{ME}+\mathrm{SA}-\mathrm{SI}$ & 20 \\
\hline $\begin{array}{l}\text { Rumex dentatus L. } \\
\text { Portulacaceae }\end{array}$ & Ann & Th & $\mathrm{ME}+\mathrm{IR}-\mathrm{TR}+\mathrm{ER}-\mathrm{SR}$ & 20 \\
\hline $\begin{array}{l}\text { Portulaca oleracea L. } \\
\text { Scrophulariaceae }\end{array}$ & Ann & Th & COSM & 45 \\
\hline $\begin{array}{l}\text { Veronica polita } \mathrm{Fr} . \\
\text { Solanaceae }\end{array}$ & Ann & $\mathrm{H}$ & ME+IR-TR+ER-SR & 5 \\
\hline $\begin{array}{l}\text { Solanum nigrum } \mathrm{L} . \\
\text { Tiliaceae }\end{array}$ & Ann & Th & COSM & 15 \\
\hline $\begin{array}{l}\text { Corchorus olitorius L. } \\
\text { Urticaceae }\end{array}$ & Ann & Th & PAN & 15 \\
\hline $\begin{array}{l}\text { Urtica urens L. } \\
\text { Zygophyllaceae }\end{array}$ & Ann & Th & ME+IR-TR+ER-SR & 10 \\
\hline Tribulus terrestris $\mathrm{L}$. & Ann & $\mathrm{H}$ & COSM & 5 \\
\hline
\end{tabular}


The invasive species Commelina benghalensis $\mathbf{L}$.

Table (2): Mean importance values of the Commelina benghalensis and its associated species in the three studied habitats.

\begin{tabular}{|c|c|c|c|}
\hline \multirow{2}{*}{ Species } & \multicolumn{3}{|c|}{ Habitat } \\
\hline & MO & MN & $\mathbf{C F}$ \\
\hline Commelina benghalensis $\mathbf{L}$. & 65.8 & 47.2 & 98.8 \\
\hline Euphorbia heterophylla $\mathrm{L}$. & 10.5 & 37.4 & 10.74 \\
\hline Cyperus rotundus $\mathrm{L}$. & 8.56 & 1.6 & 13.6 \\
\hline Dactyloctenium aegyptium (L.)Willd. & 7.08 & 3.87 & 10.44 \\
\hline Digitaria sangunalis (L.) Scop. & 11.3 & 2.90 & 5.9 \\
\hline Setaria verticillata (L.) P. Beauv. & 1.97 & 1.43 & 8.1 \\
\hline Bidens pilosa $\mathrm{L}$. & 8.06 & 10.1 & - \\
\hline Amaranthus hybridus L. & 5.16 & 2.57 & - \\
\hline Cenchrus biflorus Roxb. & 1.92 & 1.13 & - \\
\hline Cynanchum acutum $\mathrm{L}$. & 0.57 & 3.53 & - \\
\hline Echinochloa colona $(\mathrm{L}$.$) Link$ & 2.13 & 1.00 & - \\
\hline Erucastrum arabicum Fisch.\&C.A. Mey. & 1.71 & 2.03 & - \\
\hline Galinsoga parviflora Cav. & 37.1 & - & 1.2 \\
\hline Portulaca oleracea $\mathbf{L}$. & 2.31 & - & 18.5 \\
\hline Trianthema portulacastrum $\mathbf{L}$. & 1.78 & - & 6.77 \\
\hline Gynandropsis gynandra (L.) Briq. & 1.08 & - & 6.27 \\
\hline Chenopodum murale $\mathrm{L}$. & 7.91 & - & 3.51 \\
\hline Convolvulus arvensis $\mathrm{L}$. & 0.14 & - & 1.03 \\
\hline Sonchus oleraceus $\mathbf{L}$. & 4.92 & - & 1.41 \\
\hline Rumex dentatus $\mathrm{L}$. & 5.68 & - & 0.4 \\
\hline Urtica urens $\mathbf{L}$. & 0.19 & - & 0.57 \\
\hline Malva parviflora $\mathbf{L}$. & 1.2 & - & 1.09 \\
\hline Cynodon dactylon (L.) Pers. & - & 20.7 & 2.73 \\
\hline Emex spinosa (L.) Campd. & - & 6.3 & 3.5 \\
\hline Corchorus olitorius $\mathbf{L}$. & - & 4.5 & 1.61 \\
\hline Euphorbia peplus $\mathbf{L}$. & 4.18 & - & - \\
\hline Solanum nigrum $\mathrm{L}$. & 2.1 & - & - \\
\hline Lamium amplexicaule $\mathbf{L}$. & 1.82 & - & - \\
\hline Eluesine indica (L.) Gaerth. & 1.66 & - & - \\
\hline Brachiaria deflexa (Schumach.) Robyns & 1.38 & - & - \\
\hline Cronopus didymus (L.) Sm & 0.74 & - & - \\
\hline Amaranthus lividus L. & 0.73 & - & - \\
\hline Veronica polita $\mathrm{Fr}$. & 0.79 & - & - \\
\hline Stellaria pallida (Dumort.) Murb. & 0.69 & - & - \\
\hline Tribulus terrestris $\mathbf{L}$. & 0.58 & - & - \\
\hline Ipomea obscura (L.) Ker Gawl. & 0.38 & - & - \\
\hline Eclipta prostrata (L.) & 0.27 & - & - \\
\hline Capsella bursa-pastoris (L.)Medik & 0.24 & - & - \\
\hline Ipomea hederacea Jacq. & - & 32.1 & - \\
\hline Sida alba $\mathbf{L}$. & - & 5.83 & - \\
\hline Euphorbia hirta $\mathbf{L}$. & - & 5.8 & - \\
\hline Xanthium strumarium $\mathrm{L}$. & - & 4.87 & - \\
\hline Euphorbia helioscopia L. & - & 2.93 & - \\
\hline Conyza bonariensis (L.) Cronquist & - & 2.27 & - \\
\hline Brassica tournefortii Gouan. & - & 1.43 & - \\
\hline Bromus catherticus Vahl & - & - & 0.67 \\
\hline Chenopodium album $\mathbf{L}$. & - & - & 1.59 \\
\hline
\end{tabular}

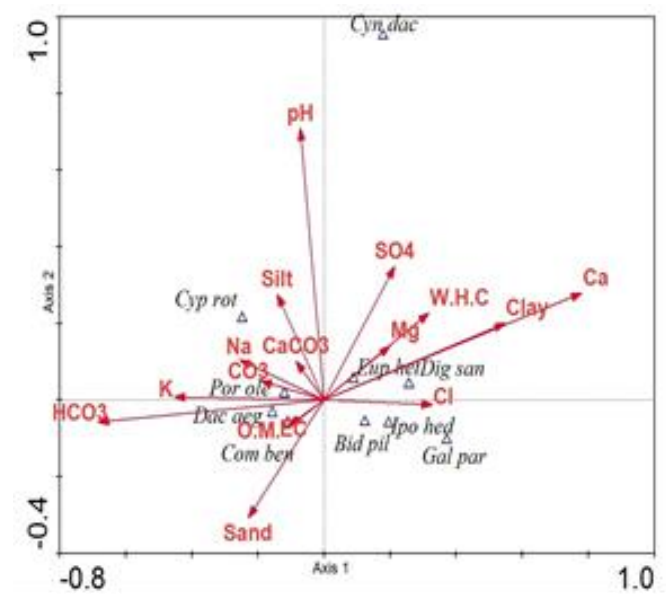

Figure (5): Biplot of CCA showing the relationships between Commelina benghalensis and its common associated species with the soil variables in the three studied habitats. Species names are abbreviated to the first three letters of genus and species. 
Abd El-Hamid H. A. \& El Bous M. M.

Table (3): Soil characteristics of the habitats supporting Commelina benghalensis community

\begin{tabular}{|c|c|c|c|c|c|}
\hline \multirow{2}{*}{ Soil variable } & \multicolumn{3}{|c|}{ Habitat } & \multirow{2}{*}{ Mean } & \multirow{2}{*}{ F- ratio } \\
\hline & MO & MN & $\mathbf{C F}$ & & \\
\hline Sand & $79.09^{b} \pm 0.67$ & $74.40^{\mathrm{a}} \pm 0.4$ & $77.71^{\mathrm{ab}} \pm 1.64$ & 77.09 & $2.82^{n s}$ \\
\hline Silt & $7.87^{\mathrm{a}} \pm 0.32$ & $9.33^{\mathrm{a}} \pm 0.44$ & $9.59^{\mathrm{a}} \pm 1.24$ & 8.69 & $1.54^{n s}$ \\
\hline Clay & $13.04^{\mathrm{a}} \pm 0.54$ & $16.27^{b} \pm 0.25$ & $12.70^{\mathrm{a}} \pm 1.76$ & 13.41 & $5.62^{* *}$ \\
\hline W.H.C \% & $45.80^{\mathrm{a}} \pm 1.90$ & $54.00^{\mathrm{b}} \pm 2.80$ & $49.37^{\mathrm{ab}} \pm 1.88$ & 48.28 & $2.74^{n s}$ \\
\hline O.M $\%$ & $1.4^{\mathrm{b}} \pm 0.94$ & $0.79^{a} \pm 0.11$ & $1.06^{\mathrm{ab}} \pm 0.12$ & 1.19 & $5.87^{* *}$ \\
\hline $\mathrm{CaCO}_{3} \%$ & $1.45^{\mathrm{a}} \pm 0.17$ & $1.94^{\mathrm{a}} \pm 0.24$ & $1.77^{\mathrm{a}} \pm 0.13$ & 1.64 & $1.76^{n s}$ \\
\hline pH & $8.39^{\mathrm{a}} \pm 0.02$ & $8.55^{\mathrm{a}} \pm 0.16$ & $8.50^{\mathrm{a}} \pm 0.07$ & 8.45 & $1.55^{n s}$ \\
\hline EC (ms/cm) & $0.84^{\mathrm{a}} \pm 0.05$ & $0.65^{\mathrm{a}} \pm 0.02$ & $0.82^{\mathrm{a}} \pm 0.07$ & 0.81 & $1.61^{n s}$ \\
\hline $\mathrm{CO}_{3}^{--} \mathrm{ppm}$ & $0.48^{\mathrm{a}} \pm 0.48$ & $0.00^{\mathrm{a}} \pm 0.00$ & $1.71^{\mathrm{a}} \pm 1.36$ & 0.84 & $0.76^{n s}$ \\
\hline $\mathrm{HCO}^{-} \mathrm{ppm}$ & $94.69^{a} \pm 5.20$ & $95.1^{\mathrm{a}} \pm 4.87$ & $105.24 \pm 3.47$ & 98.45 & $1.37^{n s}$ \\
\hline $\mathrm{Cl}^{-} \quad$ ppm & $56.8^{a} \pm 7.39$ & $47.37^{\mathrm{a}} \pm 5.93$ & $53.29^{\mathrm{a}} \pm 8.66$ & 54.2 & $0.22^{n s}$ \\
\hline SO- $\mathbf{p p m}^{-}$ & $350.41^{\mathrm{ab}} \pm 85.04$ & $648.33^{\mathrm{b}} \pm 163.97$ & $172.83^{\mathrm{a}} \pm 62.36$ & 332.95 & $4.19^{*}$ \\
\hline $\mathrm{Ca}^{++}(\mathrm{mg100g-1})$ & $580.00^{\mathrm{ab}} \pm 17.95$ & $626.67^{b} \pm 27.28$ & $547.14^{\mathrm{a}} \pm 13.58$ & 575.5 & $2.82^{n s}$ \\
\hline $\mathrm{Mg}^{++}(\mathrm{mg100g-1})$ & $24.71^{\mathrm{a}} \pm 0.59$ & $24.00^{\mathrm{a}} \pm 1.48$ & $24.24^{\mathrm{a}} \pm 0.62$ & 24.43 & $0.22^{n s}$ \\
\hline $\mathrm{Na}^{+} \quad(\mathrm{mg} 100 \mathrm{~g}-1)$ & $3.64^{\mathrm{a}} \pm 0.15$ & $3.60^{\mathrm{a}} \pm 0.23$ & $4.43^{\mathrm{b}} \pm 0.26$ & 3.91 & $4.65^{*}$ \\
\hline $\mathrm{K}^{+} \quad(\mathrm{mg100g-1})$ & $9.08^{\mathrm{a}} \pm 0.31$ & $8.47^{\mathrm{a}} \pm 0.37$ & $9.46^{\mathrm{a}} \pm 0.58$ & 9.12 & $0.75^{n s}$ \\
\hline
\end{tabular}

Variations in shoot length, leaf area, phyto-mass and succulence of $\boldsymbol{C}$. benghalensis in the studied habitats

The variations in shoot length, leaf area, phytomass and succulence of $C$. benghalensis in the studied habitats were shown in table (4). The highest $C$. benghalensis shoot length $(78.29 \mathrm{~cm})$ and phytomass $(306.24 \mathrm{~g}$ $\mathrm{DW} / \mathrm{m}^{2}$ ) were recorded in the crop field's habitat $(\mathrm{CF})$, while the lowest value of shoot length $(56.66 \mathrm{~cm})$ and phytomass $\left(28.97 \mathrm{~g} \mathrm{DW} / \mathrm{m}^{2}\right)$ were in newly cultivated mango orchards habitat (MN). On the other hand, the highest succulence (8.6) and leaf area $\left(22.04 \mathrm{~cm}^{2}\right.$ leaf-1 $)$ were recorded in old cultivated mango orchards habitat (MO), but the lowest value of succulence (6.14) and the mean leaf surface area $\left(14.59 \mathrm{~cm}^{2}\right.$ leaf- 1$)$ were in crop fields habitat (FC).

Table (4): Mean \pm standard errors of the shoot length, leaf area, phytomass and succulence of Commelina benghalensis in the three studied habitats.

\begin{tabular}{llll}
\hline \hline \multirow{2}{*}{ Variable } & \multicolumn{3}{c}{ Habitat } \\
\cline { 2 - 4 } & \multicolumn{1}{c}{ MO } & \multicolumn{1}{c}{ MN } & \multicolumn{1}{c}{ CF } \\
\hline Shoot length $(\mathbf{c m})$ & $63.6 \pm 6.62$ & $56.66 \pm 1.2$ & $78.29 \pm 5.42$ \\
Leaf area $\left(\mathbf{c m}^{\mathbf{2} \text { leaf-1 }}\right)$ & $22.04 \pm 1.82$ & $15.70 \pm 1.26$ & $14.59 \pm 1.56$ \\
Phytomass $\left(\mathbf{g ~ D W} . \mathbf{m}^{2}\right)$ & $185.26 \pm 66.34$ & $28.97 \pm 14.32$ & $306.24 \pm 110.53$ \\
Succulence & $8.60 \pm 0.71$ & $8.33 \pm 0.19$ & $6.14 \pm 0.69$ \\
\hline \hline Abbreviations: (MO) old cultivated mango orchards, (MN) newly cultivated mango orchards, (CF) crop fields
\end{tabular}

\section{Phytochemical results}

\section{Proximate composition and mineral content}

The moisture content of fresh aerial parts based on wet basis of $C$. benghalensis $\left(\mathrm{M}_{\mathrm{wet}}\right)$ was found to be 89.6 $\pm 0.17 \%$; after oven drying, it still contained a moisture level with a mean value of 10.39 (Table 5).

Proximate analyses of the studied plants shown in table (5) shows highest ash content $(31.62 \pm 2.1)$ and relatively high crude protein $(15.56)$, whereas the total carbohydrates was (13.19). Crude fiber and fat content were 1.86 and 29.24 respectively.

Table (5): Proximate composition of aerial parts of Commelina benghalensis

\begin{tabular}{lc}
\hline \multicolumn{1}{c}{ Analysis } & Mean \pm SD \\
\cline { 1 - 2 } Proximate analysis (g/100 g DW) & \\
\hline Moisture content & $10.39 \pm 0.09$ \\
Crude Protein & $15.56 \pm 0.07$ \\
Crude fiber & $1.86 \pm 0.3$ \\
Crude fat content & $29.24 \pm 1.3$ \\
Carbohydrates & $13.19 \pm 2.7$ \\
Total ash & $31.62 \pm 2.1$ \\
Nutritive value (Cal/100 gm) & 374.12 \\
\hline \hline Each value represents the mean \pm SD of three determinations on dry weight \\
(DW) basis
\end{tabular}

\section{Mineral composition}

Table (6) showed the values of the mineral compositions in milligram per $100 \mathrm{~g}$ dry weight with varying amounts of minerals such as potassium, iron, magnesium, phosphorus, calcium, manganese, zinc and copper. The highest mineral was Calcium with $1830 \mathrm{mg} / 100 \mathrm{gm}$, while the lowest value was $40 \mathrm{mg} / 100 \mathrm{gm}$ dry weight for copper.

Table (6): Mineral composition of Commelina benghalensis.

\begin{tabular}{ll}
\hline \hline \multicolumn{2}{c}{ Analysis } \\
\hline Minerals (mg/100 g DW) & Mean \pm SD \\
\hline Phosphorus & $469.9 \pm 0.76$ \\
Potassium & $1399.9 \pm 3.22$ \\
Calcium & $1830 \pm 9.87$ \\
Magnesium & $290 \pm 1.77$ \\
Iron & $1080 \pm 15.45$ \\
Manganese & $380 \pm 0.65$ \\
Zinc & $460 \pm 0.41$ \\
Copper & $40 \pm .03$ \\
\hline \hline Values means + SD are calculated as milligram per 100-gram dry weight \\
(DW) that analyzed individually in triplicate \\
\hline
\end{tabular}

\section{Phytochemical screening}

The results of preliminary phytochemical screening are shown in table (7) indicates the presence of saponins, 
The invasive species Commelina benghalensis $\mathbf{L}$.

tannins, flavonoids, glycosides and phytosterols and

absence of alkaloids and phenols.

Table (7): Preliminary phytochemical screening of Commelina benghalensis

\begin{tabular}{llllllll}
\hline \hline $\mathbf{2}^{\text {ry }}$ metabolites & Saponins & Tannins & Alkaloids & Flavonoids & Phenols & glycosides & phytosterols \\
\hline Result & + & + & - & + & - & + & + \\
\hline \hline
\end{tabular}

Identification of phytochemical components

The GC-MS analysis of the ethanol extract of Commelina benghalensis revealed the presence of fourteen compounds that could contribute the medicinal quality of the plant. The identification of the phytochemical compounds was confirmed based on the retention time and molecular formula. The fragmentation patterns for some of the peaks were compared with that of the library of compounds. The ethanol extract cons- tituents along with their retention time and their molecular weight are tabulated in table (8).

The GC-MS analysis revealed the presence of 14 compounds at two different retention times (3.749 and 19.91 minutes). The molecular weights of the compounds separated at the retention time of 3.749 min ranged from 142-268; while the molecular weight of the compounds separated at the retention time of $19.91 \mathrm{~min}$ ranged from 96 to 334 .

Table (8): The molecular weight, molecular formula and decided names for the compounds extracted by GC-MS analysis at two different retention times in ethanolic extract of Commelina benghalensis (Aerial parts)

\begin{tabular}{ccccl}
\hline \hline Se & RT (Min.) & M. wt & Mol. Formula & Compound Decided Name \\
\hline $\mathbf{1}$ & 19.91 & 208 & $\mathrm{C}_{11} \mathrm{H}_{12} \mathrm{O}_{4}$ & 2,3-Dimethoxycinnamic acid \\
$\mathbf{2}$ & 19.91 & 297 & $\mathrm{C}_{18} \mathrm{H}_{19} \mathrm{NO}_{3}$ & L-Proline, N-(1-naphthoyl)-, ethyl ester \\
$\mathbf{3}$ & 19.91 & 334 & $\mathrm{C}_{21} \mathrm{H}_{34} \mathrm{O}_{3}$ & Myristic acid, 4-methoxyphenyl ester \\
$\mathbf{4}$ & 19.91 & 254 & $\mathrm{C}_{14} \mathrm{H}_{22} \mathrm{O}_{4}$ & 9-Oxabicyclo(3.3.1)nonan-2 \\
$\mathbf{5}$ & 3.749 & 142 & $\mathrm{C}_{10} \mathrm{H}_{22}$ & n-Decane \\
$\mathbf{6}$ & 3.749 & 184 & $\mathrm{C}_{13} \mathrm{H}_{28}$ & Tridecane \\
$\mathbf{7}$ & 3.749 & 198 & $\mathrm{C}_{14} \mathrm{H}_{30}$ & 2-Methyltridecane \\
$\mathbf{8}$ & 3.749 & 170 & $\mathrm{C}_{12} \mathrm{H}_{26}$ & 2,2,4,6,6-PentaMethylheptane \\
$\mathbf{9}$ & 3.749 & 268 & $\mathrm{C}_{19} \mathrm{H}_{40}$ & 2,6,10,14-tetramethylpentadecane \\
$\mathbf{1 0}$ & 19.91 & 204 & $\mathrm{C}_{15} \mathrm{H}_{24}$ & Germacrene D \\
$\mathbf{1 1}$ & 19.91 & 188 & $\mathrm{C}_{11} \mathrm{H}_{24} \mathrm{O}_{2}$ & 2-Methyl-2,5-decanediol \\
$\mathbf{1 2}$ & 3.749 & 176 & $\mathrm{C}_{12} \mathrm{H}_{16} \mathrm{O}$ & 4-Methyl-1-phenylpentan-2-one \\
$\mathbf{1 3}$ & 19.91 & 192 & $\mathrm{C}_{11} \mathrm{H}_{12} \mathrm{O}_{3}$ & Myristicin \\
$\mathbf{1 4}$ & 19.91 & 96 & $\mathrm{C}_{6} \mathrm{H}_{8} \mathrm{O}$ & 2,5-Dimethylfuran \\
\hline \hline
\end{tabular}

\section{DISCUSSION}

Commelina benghalensis L. was chosen for investigation due to its vigorous and invasive (Burns, 2004) growth habit in the study area. Plant communities dominated by C.benghalensis were studied. It has been examined in morphological, anatomical, phytochemical and ecological aspects to evaluate its effectiveness and impact of different factors on its growth. The morphological traits of Commelina benghalensis L. could be significant if considered with anatomical features (Tomlinson, 1966; Gajurel and Shrestha, 2009). The results of the current study confirmed this concept. Morphogically, the most distinctive characters are its decumbent diffused habit, fibrous root with cleistogamous flower, sheathy pseudo-petiole, ovate leaf, cymes enclosed in conduplicate funnel shaped spathe, blue zygomorphic chasmogamous flower that rapidly fed out when exposed to sun light. Besides it has characteristics cleistogamous flowers, it is also distinguished by dimorphic flowers, fruit and seeds. Although the results generally agree with the flora of Egypt (Boulos, 2005), the seeds and capsules characters were not accurately defined. Capsule produced by chasmogamous flower was oblongelliptic 4.5-5.5 $\times 3 \mathrm{~mm}$; while the capsule produced by cleistogamous flowers was ellipsoid 5-7x3-4 mm splits along two valves, dehiscent, each containing 1 or 2 seeds with. Seeds dimorphic 3-5 per capsule with varying size; Aerial seeds $2.5-5 \times 1.5$ $2.5 \mathrm{~mm}$ oblong, obtuse, brown, rugose, irregularly reticulate and dorsal longitudinal ridge, hilum narrow; cleistogamous seeds bigger 3.5-5.2×1.2-2. Curved elliptic, nearly smooth, brown, ventral concave side with a central raised circular ridge and a short shallow groove extending transversely. The findings of dimorphic capsules and seeds justify the different dormancy properties (Budd et al., 1979) and vigorous growth.

Anatomical characters have utilized in resolving quite a number of taxonomic problems at both the generic and specific levels (Kadiri, 2006). The micromorphological finding reveals that the stomata are hexaparacytic due to arrangement of six subsidiary cells is circular shape, where the two lateral pairs parallel to the long axis of the pore and two polar (terminal) cells; the second lateral pair as long as the stomatal complex. This type could be described as a modification of the tetracytic type with an additional pair of lateral subsidiary cells (Cotthem, 1970). This feature matches with the findings of (Tomlinson, 1966), (Faden and Hunt, 1991) and (Butler, 2017). Regarding trichomes, the pubescent leaf and stem are of two types of uniseriate, eglandular and multicellular hairs with both pointed and hooked distal cell in accordance with Oladipo (2014). It also has onecelled papillae restricted to leaf margin. Those types of trichomes constitutes important distinguishing features that demarcate the genus Commelina (Tomlinson, 
1966). Stem anatomy can be differentiated by stomata at the same level with the epidermal cell, substomatal cavity, and outer ring of collateral vascular bundle surrounded by fiber sheath while the medullar vascular bundles are reduced to phloem and large Lysigenous lacuna. Leaf lamina thickness $(356.7 \pm 2.79)$ and large size of cells with thin wall indicates the leaf succulence. Similar anatomical traits were previously demonstrated to family Commelinaceae (Novoa et al., 2012; Novoa and Arambarri, 2016).

The floristic components of Commelina benghalensis community were 47 species belonging to 21 families. Poaceae, Asteraceae and Brassicaceae were the most important as they contribute more than $49 \%$ of the species. These leading families were reported to be the most frequent in other studies on the cultivated lands of Egypt by (Abd El-Hamid, 2005) and (Mashaly et al., 2012). Regarding the plant longevity, $89.4 \%$ of the recorded species are annuals, $(8.5 \%)$ perennials and one species biennial. The dominance of annuals could be attributed to their high reproductive capacity and ecological, morphological and genetic plasticity under high levels of disturbance (Harper, 1977). Moreover, they have the ability to produce the flowers early in their life span in order to ensure some seed production even in a year when the growing season is cut short (Sans and Masalles, 1995).

The life form spectra provide information, which may help in assessing the response of vegetation to variations in environmental factors (Ayyad and Ghabbour, 1986). The present study showed that therophytes had the highest contribution, followed by hemicryptophytes and geophytes. Therophytes represent the main floristic element in the cultivated land. Many of these have a short life cycle, which enable them to cope with the instability of the agro-ecosystems in which they occur and their ability to produce heteromorphic seeds (Abu Ziada et al., 2014) .

Chorological analysis of the floristic data in the present study revealed that the palaeotropical, Mediterranean and cosmopolitan taxa form a relatively high proportion of species. This is can be attributed to the relatively high contribution of summer annual species in the Commelina benghalensis community. Abd ElHamid (2005) reported that, most of the summer annual weeds belong to the paleotropical category, while winter weeds belong to Mediterranean and Cosmopolitan categories. The high contribution of the Mediterranean taxa in the study area agreed with the most current of weed flora of Egypt (Kosinova, 1974; El-Hadidi, 1993).

The vegetation analysis of summer crops and orchards in the study area indicated the dominance of Commelina benghalensis in the three studied habitats, while Portulaca oleracea, Galinsoga parviflora, Cyperus rotundus, Dactyloctenium aegyptium, Digitaria sanguinalis, Euphorbia heterophylla, Ipomoea hederacea and Bidens pilosa represented the most common associated species. This finding contradicts with that reported by Abd El-Hamid (2005) in the same region in which, Digitaria sanguinalis, Cynodon dactylon, Portulaca oleracea, Cyperus rotundus and Euphorbia heterophylla were the dominant species in the summer crops; while in the present study, these species are recorded as the common species associated with $C$. benghalensis. The dominance of $C$. benghalensis could be attributed to its unique biological characteristics which are represented by its fast spread as it can propagate by both sexual and vegetative, it produces aerial and subterranean seeds from its chasmogamous and cleistogamous flowers (Maheshwari, 1955). It can produce up to 1600 seeds per plant, roots readily at the nodes of creeping stems and regenerates rapidly in this fashion when broken or cut (Holm, 1977). The rate of reproduction of this plant rivals that of any agronomic weed (Webster et al., 2005). The change of dominant species could be attributed to the regular weeding, either mechanically or through the use of herbicides in summer crops which decrease the number of weed species and causes the change in weed flora, especially the dominant ones. The agricultural practices and crop plants have the major effect on the weed flora (Krupinsky et al., 2006).

Soil characteristics are considered as important environmental factors affected weed community structure and diversity (Fried and Norton, 2008; Pinke et al., 2010). The inspection of CCA ordination diagram showed that Commelina benghalensis exhibited a clear relationship with organic matter and sand fraction. These findings is rather similar with that reported by Ahmad et al. (2016) in which Commelina benghalensis is found in abundance in communities that exposed to moderate moist condition with application of irrigation and fertilizers. In addition, $C$. benghalensis is considered a rainy season weed which require moist soil condition for establishment and can survive dry condition after its establishment (Kaul et al., 2002). C. benghalensis grows well on all soil types of variable $\mathrm{pH}$ and moisture levels (Webster et al., 2005).

The shoot length and phytomass of $C$. benghalensis recorded the highest values in the crop fields $(\mathrm{CF})$. This could be attributed to high light intensity and continuous addition of fertilizers compared to mango orchards. Light directly affects growth, morphology and accumulation of biomass of plants. Under low light, growth and development of plants are retarded and rate of biomass accumulation and seed production decrease (Reich et al., 1992) and (Lambers et al., 2008). Riar et al. (2016) pointed out that, growth and competitiveness of $C$. benghalensis would primarily be influenced by fertilization in the crop system. The highest leaf area was recorded in old cultivated mango orchards (MO) with high trees and large canopy, which decrease the light intensity. This finding is incompatible with Riar et al. (2016) who concluded that shading had little effect on total leaf number and leaf area of $C$. benghalensis plant. Regnier et al. (1988) observed the greater specific leaf area and leaf area ratio under reduced light conditions with Datura stramonium L., Abutilon theophrasti Medik. and Soybean. Shade enhanced Isatis tinctorial s light-harvesting efficiency by increasing leaf area (Monaco et al., 2005).

The potential quality of Commelina benghalensis plant was investigated. The proximate analysis showed that the moisture content based on wet basis of $C$. benghalensis (Mwet) was found to be $89.6 \pm 0.17$; and 
this result with the anatomical and ecological data ensure the succulence nature of the plant. The highwater content in $C$. benghalensis makes it ideal to use as fodder. According to Squires et al. (1994) the forage species having high ash had higher palatability, also moderate moisture content makes feed more palatable.

The quality assessment of the functional properties of plant is measured by ash content (Onwuka, 2005). High level of total ash, up to $31.62 \pm 2.1 \%$ in the aerial parts of the plant indicates high inorganic components (Chioma and Omoregie, 2010). Crude fiber in plant is an indication of the level of non-digestible carbohydrate, cellulose and lignin, its low level $(1.86 \pm 0.3)$ is considered appropriate; therefore, its presence in low level can increase nutrient usage and prevent intestinal irritation (Bouba et al., 2012). As the fiber content of a forage increases, its energy content decreases (Stokes and Prostko, 1998). Furthermore, the plant is a moderate source of protein $(15.56 \pm 0.07 \mathrm{~g} / 100 \mathrm{~g})$. Kearl (1982) suggested that $11-13 \%$ level of crude protein in the diet is sufficient for maintenance and growth requirements of sheep and goats, thus the investigated crude protein value is relatively high. The results of the fat analysis indicated that the plant contains a reasonable content of fat $(29.24 \pm 1.3)$. Lipid as a good source of energy contributes to important cell processes and aids in transport of fat soluble vitamins (Pamela et al., 2005).

According to Le Houérou (1980) and Heneidy and Bidak (2003) the forage value of the consumed plant is the result of its nutritive value. It was believed that livestock will refuse eating weeds with low nutritive value, so expensive and time-consuming measures are often used for their control (Marten and Andersen, 1975). The nutritive value of aerial parts of Commelina benghalensis was $374.12 \mathrm{Cal} / 100 \mathrm{gm}$, hence, the plant can contribute to the caloric requirement of livestock.

The mineral compositions of aerial parts of C. benghalensis, illustrate that it has significant levels of macroelements (Calcium $1830 \pm 9.87$, potassium 1399.9 \pm 3.22 and phosphorus $469.9 \pm 0.76$ ). Macro minerals are required at concentrations greater than $100 \mathrm{ppm}$ and have important physiological functions of the diet and therefore, must be supplemented to livestock when forages or rations are deficient or have the incur-rect proportions of macro minerals; while trace minerals are required at concentrations less than $100 \mathrm{ppm}$ (Doxey, 1992). The ratio of $\mathrm{Ca} / \mathrm{P}(4: 1)$ was an accept-able ratio, that is between $1: 1$ and $7: 1$, as long as there is enough phosphorus (P) to meet the animal's nutritional requirements (Santos et al., 2004). Zinc (460 \pm 0.41 $\mathrm{mg} / 100 \mathrm{~g}$ ) is required for the structural integrity of enzymes where zinc metalloenzymes involved in nucleic acid and protein synthesis (Abdel-Mageed and Oehme, 1990). Copper is very vital in diet because it is involved in the proper usage of iron that was $1080 \pm$ 15.45. The potassium content was $1399.9 \pm 3.22 \mathrm{mg} / 100$ g. Potassium is very vital in regulation of water and acid-base balance in the body. Manganese (380 \pm 0.65 $\mathrm{mg} / 100 \mathrm{~g}$ ) as an essential trace element was based on measurements of reproductive parameters and has also been identified as an essential component in bone and cartilage formation and growth (Paterson and Engle,
2005). The potassium content was $290 \pm 1.77 \mathrm{mg} / 100 \mathrm{~g}$, It is vital in regulation of water and acid-base balance in the body (EFSA, 2006). These results match with the findings of Gole et al. (2013) that C. benghalensis has potential to uptake mineral.

Phytochemical examinations of aerial parts extracts revealed the presence of saponin, tannins, flavonoid, glycosides and phytosterols. These results have been established by Augustine et al. (2013) and Tadesse et al. (2016). The phytoconstituent rich ethanolic extract was elucidated using GC-MS as one of the best techniques which allows simultaneous assessment of a variety of components in the plant (Hussain and Maqbool, 2014). Among the decided compound 2,3-Dimethoxycinnamic acid that was previously recognized as a naturally occurring aromatic fatty acid among the members of the aromatic fatty acid class of differentiation-inducers with potential use in cancer intervention (Liu et al., 1995); Furthermore, Myristic Fatty acid also had been shown to have potent anti-cancer properties (Tatsuya et al., 2003).

Another observed compound was L-Proline, N-(1naphthoyl), ethyl ester. Zhang et al. (2016) recorded that proline amino acid is a precursor for hydroxyproline, which is used to synthesize collagen; so, this protein is used to cushion joints and repair. Germacrenes are typically produced in a number of plant species for their antimicrobial activity (Flamini et al., 2005). Previous studies on chemical investigation of C.benghalensis by GC-MS revealed the presence of fourteen different compounds; the most investigated compounds were belonging to acid group. Others suggested that this plant was a potential source of 10 pharmacologically active chemical compounds and therefore investigate its antifungal, antimicrobial and antiinflammatory activity (Cuéllar Cuéllar and Okori, 2010; Augustine et al., 2013; Kadam, 2016).

\section{CONCLUSION}

In conclusion, findings of the morphological, anatomical, mineral and proximate composition and phytochemical constituents showed that succulent invasive weed Commelina benghalensis can be considered a palatable and relatively low-cost source of nutrition for livestock, besides it could be used as a potential source of new useful drugs. Isolation of bioactive compounds and studying their biological activity are necessary for future studies. It also can be concluded from the results that Commelina benghalensis has the potential to flourish well in different habitat types and tolerate environmental stress.

\section{REFERENCES}

ABD EL-GAWAD, A. 2014. Ecology and allelopathic control of Brassica tournefortii in reclaimed areas of the Nile Delta, Egypt, Turkish Journal of Botany, 38(2), pp. 347-357. Available at: http://journals. tubitak.gov.tr/botany/abstract.htm? id=14706

ABD EL-GHANI AND ABDEL-KHALIK. 2006 
'Floristic diversity and phytogeography of the Gebel Elba National Park, south-east Egypt', journals. tubitak.gov.tr, 30, pp. 121-136. Available at: https:// journals.tubitak.gov.tr/botany/abstract.htm? id=8200

ABD EL-GHANI, M. M. 1981. Preliminary studies on the vegetation of Bahariya Oasis Egypt. M.Sc. Thes.

ABD EL-GHANI, M. M. 1985. Comparative study of the vegetation of Bahariya and Farafra Oases and the Faiyum region,Egypt. unpublishe.

ABD EL-HAMID, H. A. 2005. 'Ecological Study of Weed Vegetation and local Environment in Ismailia Governorate, Egypt., Ph.D. Thesis, Faculty of Science, Suez Canal University.

ABDEL-MAGEED, A. B., AND F.W. OEHME. 1990 'A review of the biochemical roles, toxicity and interactions of zinc, copper and iron: I. Zinc.', Veterinary and human toxicology, 32(1), pp. 34-9. Availableat: http: //www.ncbi.nlm.nih.gov /pubmed 12405577

ABU ZIADA, M.E.A., G.A. EL-SHERBENY, AND M. R. ASKAR. 2014. 'Ecology and Phytochemistry of Stinking Chamomile (Anthemis cotula L.) in', Asian Journal of Plant Sciences, 13, p. 156-163. https://www.researchgate.net/profile/Ghada El_Sherbeny/publication/277592710 Ecology and Phytochemistry of Stinking Chamomile Anthemis cotula L in Egypt/links/5656322e08ae4988a7 b377cc.pdf .

AHMAD, Z., S.M. KHAN, E.F. ABD_ALLAH, AND A.A., ALQARAWI. 2016. 'Weed species composition and distribution pattern in the maize crop under the influence of edaphic factors and farming practices: A case study from Mardan, Pakistan', Saudi Journal of Biological Sciences. Elsevier, 23(6), pp. 741-748. doi: 10.1016/J.SJBS. 2016.07. 001.

ALLEN, S. E., H.M. GRIMSHAW, AND J.A. PARKINSON. 1974. Chemical analysis of ecological materials. Oxford, UK: Blackwell Scientific Publications.

ANDERSSON, T. AND P. MILBERG. 1998. Weed flora and the relative importance of site, crop, crop rotation, and nitrogen', Weed Sci., (46): p. 30-38. Available at: http://www.jstor.org/stable/4046005.

ANDREASEN, T.N. AND I. SKOVGAARD. 2009. Crop and soil factors of importance for the distribution of plant species on arable fields in Denmark, Agr. Ecosystems Envir, (133), p. 61-67. Available at:https://www.sciencedirect.com/science/ article/pii/S016788090900142X

AOAC, 1990. Official Methods of Analysis, 15th ed. Association of Official Analytical Chemists, Washin-gton, DC. Washington, DC.

AUGUSTINE, B., S.K. TIWARI, M. LAHKOR, S. DASH, AND P. SAMUDRALA. 2013. Preliminary phytochemical, toxicity and anti-inflammatory evaluation of Commelina benghalensis, International Journal of Green Pharmacy, 7(3), p. 201. doi: 10.4103/0973-8258.120211.

AYYAD, M. A., AND R.E.M. EL-GHAREEH. 1982. Salt marsh vegetation of the Western Mediterranean desert of Egypt, Vegetatio, 49(1), pp. 3-19. doi: 10.1007/BF00051557.
AYYAD, M.A., AND S.I. GHABBOUR. 1986. Hot deserts of Egypt and the Sudan, Ecosystems of the world., 12, pp. 149-202. Available at: http://cat. inist.fr/?aModele $=$ afficheN\&cpsidt=8589823 (Accessed: 17 March 2018).

BARUAH, T. AND H. BARTHAKUR. 1997. A Textook of Soil Chemical Analysis. Vikash, New Delhi.

BOUBA, A.A., N.Y. NJINTANG, H.S. FOYET, J.SCHER, D.MONTET, AND C.M.F. MBOFUNG. 2012. Proximate Composition, Mineral and Vitamin Content of Some Wild Plants Used as Spices in Cameroon', Food and Nutrition Sciences, 03(04), pp. 423-432. doi: 10.4236/fns.2012.34061.

BOULOS, L. 1999. Flora of Egypt. Al Hadara Pub. Availableat:http://kbd.kew.org/kbd/detailed result. do? id=351365 (Accessed: 30 October 2017).

BOULOS, L. 2002. 'Flora of Egypt: volume 3. (Verbenaceae - Compositae), kbd.kew.org, 373p.illu.Available at: http://kbd.kew.org/kbd/detailed result.do? id $=351365$

BOULOS, L. 2005. Flora of egypt. Available at: http://www.egyptologyforum.org/THOE/Heritage of Egypt 5 arabic.pdf (Accessed: 21 January 2018).

BOUYOUCOS, G.J. 1962. 'Hydrometer Method Improved for Making Particle Size Analyses of Soils1', Agronomy Journal. American Society of Agronomy, 54(5), p. 464. doi: 10.2134/agronj $1962.00021962005400050028 x$.

BUDD, G.D., P.E.L. Thomas, J.C.S. Allison. 1979. Vegetation regeneration, depth of germination and seed dormancy in Commelina benghalensis L., cabdirect.org. Available at: https:// www. cabdirect. org /cabdirect /abstract/ 19802331730 (Accessed: 19 December 2017).

BURNS, J.H. 2004. A comparison of invasive and noninvasive dayflowers (Commelinaceae) across experimental nutrient and water gradients', Diversity and Distributions, 10(5-6), pp. 387-397. doi: 10.1111/j. 1366-9516.2004.00105.x.

BUTLER, L.E.E.A. 2017. A Classification of', (July 1970), pp. 235-246.

CANFIELD, R. 1941. Application of the line interception method in sampling range vegetation, Journal of forestry, 39, p. 288-394. Available at: https://academic.oup.com/jof/article-abstract /39 /4 /388/4706387.

CHIOMA, V. AND H. OMOREGIE. 2010. Pharmacognostic and Phytochemical Analysis of Commelina benghalensis, Ethnobotanical Leaflets, 14 (Burkill 2000), pp. 610-15.

COTTHEM, W.R.J. 1970. A classification of stomatal types', Botanical Journal of the Linnean Society. Oxford University Press, 63(3), pp. 235-246. doi: 10.1111/j.1095-8339.1970.tb02321.x.

CUÉLLAR CUÉLLAR, A., AND D.O.OKORI. 2010. Preliminary Phytochemical and Antimicrobial Evaluation of the Fresh and Dried Whole Plant Extracts from Commelina benghalensis, Revista Colombiana de Ciencia Animal, 2(1), pp. 104-116.

CUTLER, D.F., T. BOTHA, D.W. STEVENSON. 2008. Plant anatomy: an applied approach, Turrialba 
(IICA)v. 37(1) p. 77-84. Blackwell Pub.

DAEHLER, C.C. 1998. The taxonomic distribution of invasive angiosperm plants: Ecological insights and comparison to agricultural weeds, Biological Conservation, 84(2), pp. 167-180.

DEL PERO MARTÍNEZ, M.A., AND T. SWAIN.1985. Flavonoids and chemotaxonomy of the Commelinaceae, Biochemical Systematics and Ecology, 13 (4),pp.391-402.doi:10.1016/0305-1978(85)90083-3.

DOXEY, D.L. 1992. Minerals in animal and human nutrition', Tropical Animal Health and Production. Kluwer Academic Publishers, 24(4), pp. 241-241. doi: 10.1007/BF02356753.

EFSA. 2006. Tolerable Upper Intake Levels for Vitamins and Minerals, Scientific Committee on Food Scientific Panel on Dietetic Products, Nutrition and Allergies.

EL-HADIDI, M. N. 1993. Natural Vegetation. In The Agriculture of Egypt. (Graig, G.M.cd.), Oxford University Press, 3, p. 39-62.

FADEN, R.B. AND D.R. HUNT. 1991. The classification of the Commelinaceae, Taxon. JSTOR, pp. 19-31.

FAO-UNESCO. 1964 .Guidelines for soil profile description, Rome, FAO.

FERRELL, J., G.E. MACDONALD, AND B.J. BRECKE. 2006. Tropical Spiderwort (Commelina benghalensis L.), Identification and Control 1, Control, pp. 2-4.

FLAMINI, G., P.L. CIONI, AND I. MORELLI. 2005. Composition of the essential oils and in vivo emission of volatiles of four Lamium species from Italy: L. purpureum, L. hybridum, L. bifidum and L. amplexicaule, Food Chemistry, 91(1), pp. 63-68. doi: 10.1016/j.foodchem.2004.05.047.

FRIED, G., AND L. NORTON. 2008. Environmental and management factors determining weed species composition and diversity in France, Agric. Ecosystem. Environ, 128, p. 68-76. Available at: https://www.sciencedirect.com/science/article/pii/S0 167880908001424

GAJUREL, J.P., AND K.K. SHRESTHA. 2009. Taxonomy of the genus Commelina Plum.exL. (Commelinaceae ) in Nepal, pp. 25-31.

GOLE, K.A., D.A. KADAM, S.N. JADHAV, AND V.T. APARADH. 2013. Mineral Uptake Potential of Commelina Bengalensis, Cyanotis Cerifolia and Zebrina Pendula. International Journal of Pharmaceutical Research and Allied Sciences, 2(2), pp. 70International Journal of Institutional Pharmacy and Life Sciences, 6(3), pp. 505-512.

KADIRI, A. 2006. Comparative foliar micromorphological characters-Google Scholar, Bulletin of Pure and Applied Sciences - Botany, 25 (1), pp. 21-26. Available at: https:// scholar. google.com /scholar? $\mathrm{hl}=\mathrm{en}$ and as_sdt $=0 \% 2 \mathrm{C} 5$ and $\mathrm{q}=$ Comparative + foliar + micromorphological + characters + of + the + species +of+Portulaceaceae+in+Nigeria and btnG.

KAUL, V., N. SHARMA, AND A.K. KOUL. 2002. 'Reproductive effort and sex allocation strategy in Commelina benghalensis L., a common monsoon weed, Botanical Journal of the Linnean Society.
75. Available at: http:// search. ebscohost. com / login. aspx? direct $=$ true and $\mathrm{db}=\mathrm{a} 9 \mathrm{~h}$ and $\mathrm{AN}=93465447$ and site $=$ ehost-live and scope $=$ site.

GOOD, R. 1974. The geography of the flowering plants ( $4^{\text {th }}$ ed.). LongmanHARBORNE, A. 1998. Phytochemical methods a guide to modern techniques of plantanalysis.Availableat:https://www. google.com/ books? hl=en and $\mathrm{lr}=$ and $\mathrm{id}=2 \mathrm{yvqeRtE} 8 \mathrm{CwC}$ and oi=fndand pg=PR7 and dq=methods+of + phytochemical+screening and ots $=$ xzbhS6TrS6 and sig $=\mathrm{RX}$ 2lPAtDgH8bwiFsjH8 jEcRpmI.

HARBORNE, J.B. 1984. Methods of Plant Analysis, in Phytochemical Methods. Dordrecht: Springer Netherlands, pp. 1-36. doi: 10.1007/978-94-0095570-7_1.

HARPER, J. 1977. Population biology of plants, London: Academic Press. doi: 10.1016/03043746(79)90009-X.

HENEIDY, S.Z. 2010. Plant Atlas The Botanic Garden (Alex)' Faculty of Science, Alexandria University. Monsha'a Al-Maaref, Alexandria.

HENEIDY, Z. SELIM AND L.M. BIDAK. 2003. Association Between Calcium Oxalate Crystals and Potential Palatability of Some Range Plant Species in the Mediterranean Coastal Region, Egypt, Bull.Fac. Sci., Assiut Univ., 31((1-D)), pp. 151-163.

HOLM, L.G., D.L. PLUCKNETT, J.V. PANCHO, AND J.P. HERBERGER. 1977. The world's worst weeds. University Press.

HOLM, L.G. 1977. The World's Worst Weeds. Honolulu: University Press of Hawaii.

HUSSAIN, S.Z., AND K. MAQBOOL. 2014. GC-MS: Principle, Technique and its application in Food Science, Int J Curr Sci, 13, pp. 116-126.

IBRAHIM, L.M.N. 2017. Ecological and Phytochemical Studies on Neem Plant (Azadirachta indica), Ismailia, Egypt. in M.Sc. Thesis, Faculty of Science, Suez Canal University.

ISAAC, W.A., Z. GAO, AND M. LI. 2013. Managing Commelina species: Prospects and limitations, Herbicides-Current Research and Case Studies in Use.

JOHANSEN, D.A. 1940. Plant microtechnique. McGraw-Hill Book Company, Inc: London; 530p.

JOULAIN, D. AND W.A. KÖNIG. 1998. The atlas of spectral data of sesquiterpene hydrocarbons. EBVerlag.

KADAM, K.M. 2016. ROLES OF COMMELINA BENGHALENSIS IN MEDICINE-A REVIEW, Oxford University Press, 140(4), pp. 403-413. doi: 10.1046/j.1095-8339.2002.00082.x.

KEARL, L. 1982. Nutrient requirements of ruminants in developing countries., Utah Agricultural Experimental Station, Logan, Utah, USA. Available at: https://www.cabdirect.org/cabdirect/abstract/198307 44565

KEMP, C.D. 1960. 'Methods of Estimating the Leaf Area of Grasses from Linear Measurements, Annals of Botany. Oxford University Press, 24(4), pp. 491499. doi: 10.1093/oxfordjournals.aob.a083723.

KHEDR, A.A. AND A.K. HEGAZY. 1998. Ecology of the rampant weed Nymphaea lotus L. Willdenow in 
natural and ricefield habitats of the Nile delta, Egypt, Hydrobiologia, 386, p. 119-129. Available at:http://www.springerlink.com/index/HWR8350G1 85RJ860.pdf (Accessed: 25 January 2018).

KOSINOVA, J. 1974. Studies on the weed flora of cultivated land in Egypt. 4-Mediterranean and tropical elements, Candollea, 29: 281-295., (29), pp. 281-295. Available at: http://agris.fao.org/agrissearch/search.do? recordID=US201302712495

KRUPINSKY, J.M., S.D. MERRILL, J.D. HANSON, D.L. TANAKA, M.A. LIEBIG. 2006. Crop sequence effects of 10 crops in the northern Great Plains, Agricultural Systems, 88(2-3), pp. 227-254. doi: 10.1016 / j.agsy.2005.03.011.

LAMBERS, H., F.S. CHAPIN, AND T.L. PONS. 2008. 'Photosynthesis, in Plant Physiological Ecology. New York, NY: Springer New York, pp. 11-99. doi: 10.1007/978-0-387-78341-3_2.

LANYASUNYA, T.P., H. WANG, S.T. KARIUKI, E.A. MUKISIRA, S.A. ABDULRAZAK, N.K. KIBITOK, J.O. ONDIEK. 2008. The potential of Commelina benghalensis as a forage for ruminants', Animal Feed Science and Technology. Elsevier, 144(3-4), pp. 185-195. doi: 10.1016 j. anifeedsci. 2007.10.009.

LE HOUÉROU, H.N. 1980. Chemical composition and nutritive value of browse in tropical West Africa, Browse in Africa. ILCA, Addis Ababa. Available at: https: //books. google.com /books? hl=en and lr=and id $=$ f8wzMTZzPZYC and oi $=$ fnd and pg =PA261 anddq $=$ Chemical+composition+and+nutritive+value +of+browse+in+tropical+West+Africa and ots = OsyZ-Jbl3V and sig=fFCL0J_1kvI4NfL5tX9YBi9 fdGQ (Accessed: 13 March 2018).

LEWIS, W.M., AND J.J. GREEN. 1995. Weed management, in Weed management.In: Production and utilization of pastures and forages. Raleigh (NC): North Carolina State University, North Carolina Agricultural Research Service, p. Technical bulletin 305.

LIU, L., W.R. HUDGINS, S. SHACK, M.Q. YIN, D. SAMID. 1995. Cinnamic acid: a natural product with potential use in cancer intervention. International journal of cancer, 62(3), pp. 345-50. Availableat:http://www.ncbi.nlm.nih.gov/pubmed/762887 7(Accessed: 7 December 2017).

MAHESHWARI, P. 1955. Floral dimorphism in Commelina forskalaei Vahl. and C. benghalensis L, Phytomorphology, 5, pp. 413-422. Available at: https://ci.nii.ac.jp/naid/10018297371/.

MARTEN, G.C. AND R.N. ANDERSEN. 1975. Forage Nutritive Value and Palatability of 12 Common Annual Weeds1, Crop Science. Crop Science Society of America, 15(6), p. 821. doi: 10.2135/ cropsci1975.0011183X001500060024x.

MASHALY, I., S.A. Al-BARATI, A. ABD ELGAWAD. 2012. Weed plant communities in the Nile Delta of Egypt. III, Egyptian Journal of Botany, 52(1), p. 1-26.

MBAZIMA, V.G. 2009. The Effects of Crude Methanolic Extract of Commelina benghalensis Linn on the Expression of Apoptotic and Cell Division Cycle
Genes in Jurkat T and Wil-2 NSCancer Cell Lines.' University of Limpopo (Turfloop Campus). Available at:http: // ulspace.ul.ac.za / handle / 10386 /937.

MOKGOTHO, M. 2009. Isolation and Characterisation of Bioactive Compounds from Commelina benghalensis Linn: Biological activity analysis of extracts against Wil-2 NS lymphoma. Available at:http: // 196.21.218.27/bitstream/handle/10386/941/mokgoth o m_2009. pdf ? sequence $=1$ and is Allowed $=y$.

MONACO, T.A., D.A. JOHNSON, AND J.E. CREECH. 2005. Morphological and physiological responses of the invasive weed Isatis tinctoria to contrasting light, soil-nitrogen and water, Weed Research, 45(6), pp. 460-466. doi: 10.1111/j.1365-3180.2005.00480.x.

MUCINA, L. 1997. Classification of vegetation: Past, present and future, Journal of Vegetation Science, 8(6), pp. 751-760. doi: 10.2307/3237019.

NOVOA, M.C. AND A.M. ARAMBARRI. 2016. Importance of anatomical leaf-blade features for the characterization of medicinal Commelinaceae in the Rio de la Plata area (Argentina). Boletín de la Sociedad Argentina de Botánica,Bot.51(3), pp. 419427.

NOVOA, M.C., M.N. COLARES, AND A.M. ARAMBARRI. 2012. Anatomy of monocotyledons: stems and rhizomes of land herbs used as medicinal in the Río de La Plata area (Argentina), Bonplandia, 21(2), pp. 149-158.Available at: http: //ibone. unne.edu.ar/objetos/uploads/documentos/bonplandia /public/21_2/149-157.pdf.

OLADIPO, A.A. 2014. Foliar epidermal morphology of the genera, 16(2), pp. 219-225.

ONWUKA, G.I. 2005. Food Analysis and Instrumentation; Theory and Practice.

PAMELA, C., A. RICHARD, AND R. DENISE. 2005. 'Lippincotts illustrated reviews Biochemistry, Philadelphia, 8, pp. 335-338.

PATERSON, J.A., AND T.E. ENGLE. 2005. Trace mineral nutrition in beef cattle, University of Tennessee Department of Animal Science Nutrition Conference, (1992), pp. 1-22.

PETERS, K., L. BREITSAMETER, AND B. GEROWITT. 2014. Impact of climate change on weeds in agriculture: a review, Agronomy for Sustainable Development, 34(4), pp. 707-721. doi: 10.1007/s 13593-014-0245-2.

PIERCE, W.C., E.L. HAENISCH, AND W.C. SAWPIERCE, E.L. HAENISCH, AND D.T. SAW YER, 1958. Quantitative Analysis. Wiley Toppen, Tokyo.

PINKE, G., R. PÁL, AND Z. BOTTA-DUKÁT. 2010. Effects of environmental factors on weed species composition of cereal and stubble fields in western Hungary, Central European Journal of Biology, 5(2), pp. 283-292. doi: 10.2478/s11535-009-0079-0.

PIPER, C. 1942. Soil and plant analysis. Intersience Publishers, Inc. New York. Available at: http:// krishikosh.egranth.ac.in/bitstream/1/2034372/1/CIF E-107.pdf.

RAQUIBUL HASAN, S.M., S. HOSSAIN, M. JAMILA, AND M.E. MAZUMDER. 2010. Analgesic activity of the different fractions of the aerial parts of Commelina benghalensis Linn, Interna- 
tional Journal of Pharmacology, 6(1), pp. 63-67. doi: 10.3923/ijp.2010.63.67.

RAUNKIAER, C. 1934. The life forms of plants and statistical plant geography;Translated by Carter Fausboll and Tansley, cabdirect.org, (Oxford Univ. Press, London.). Available at: https: //www. cabdirect.org/cabdirect/abstract/19340 701044.

REGNIER, E.E., M.E. SALVUCCI, AND E.W. STOLLER. 1988. 'Photosynthesis and Growth Responses to Irradiance in Soybean (\&lt;span class=and quot; italic and quot; and gt; Glycine max and lt; span and gt;) and Three Broadleaf Weeds', Weed Science. Cambridge University Press, 36(4), pp. 487-496. doi: 10.1017/s004317450007524x.

REICH, P.B., M.B. WALTERS, AND D.S. ELLSWORTH. 1992. 'Leaf Life-Span in Relation to Leaf, Plant, and Stand Characteristics among Diverse Ecosystems, Ecological Monographs, 62(3), pp. 365-392. doi: 10.2307/2937116.

REVEAL, J.L., AND M.W. CHASE. 2011. APG III: Bibliographical information and synonymy of Magnoliidae, Phytotaxa. Oxford University Press, 19(2), pp. 71-134. doi: 10.1111/j.1095-8339.2009. 00996.x.

RIAR, M.K., D.S. CARLEY, C. ZHANG, M.S. SCHROEDER-MORENO, D.L. JORDAN, T.M. WEBSTER, AND T.W. RUFTY. 2016. Environmental Influences on Growth and Reproduction of Invasive Commelina benghalensis, International Journal of Agronomy, 2016 (May). doi: 10.1155/ 2016/5679249.

ROCHA, D.C., R.A. RODELLA, AND D. MARTINS. 2007. caracterização morfológica de espécies de trapoerabalr(commelina spp.) utilizando a análise multivariadalrmorphological characterization of Wandering-Jew Species (Commelina spp.) Usingl rMultivariate Analysis, Planta Daninha, 25(4), pp. 671-678.

SANS, F.X., AND R.M. MASALLES. 1995. 'Phenological patterns in an arable land weed community related to disturbance, Weed Research, 35(5), pp. 321-332. doi: 10.1111/j.1365-3180.1995. tb01627.x.

SANTOS, B.M., J.A. DUSKY, W.M. STALL, T.A. BEWICK. 2004. Phosphorus absorption in lettuce, smooth pigweed (Amaranthus hybridus), and common purslane (Portulaca oleracea) mixtures, Weed Science, 52(3), pp. 389-394. doi: 10.1614/ WS-03-053R.

SHALTOUT, S. 2014. Ecological study on the alien species in the Egyptian flora. Available at: https: //scholar.google.com/scholar?hl=en\&as_sdt=0\%2C5 $\& q=$ Ecological+study+on+the+alien+species+in+th e+Egyptian+Flora.\&btnG= (Accessed: 12 March 2018).

SHUKLA, R., AND P. CHANDEL. 1996. Plant ecology and soil science Edited by I. Chand S, Company LTD. Ram Nagar, New Delhi.

SQUIRES, V.R. AND A.T. AYOUB. 1994. Halophytes as a resource for livestock and for rehabilitation of degraded lands: proceedings of the International Workshop on Halophytes for Reclamation of Saline Wastelands and as a Resource for Livestock, Problems and Prospects, Nairobi, Kenya, 22-27 November 1992. Kluwer Academic.

STOKES, S.R., AND E.P. PROSTKO. 1998. Methods of Forage Quality Analysis, Agriculture, p. 2.

TACKHOLM, V. 1974. Student Flora of Egypt, Cairo University, Cooperative Printing Company, Beirut.

TADESSE, S., K. GANESAN, B. KUMAR, AND S. BANU. 2016. Preliminary phytochemical screening of different solvent extracts of leaves and stems of Commelina benghalensis L. (Family: Commelinaceae), International Journal of Pharmaceutical, Chemical \& Biological Sciences, 6(1), pp. 103-107.

TATSUYA, M., J. KEIKO, K. HIROKANZU, A. YASUSHI, S. HIROYUKI, I. TAKAHIRO AND S. KIMIO. 2003. Hepatoprotective Effect of Myristicin from Nutmeg (Myristica fragrans) on Lipopolysaccharide/d-Galactosamine-Induced Liver Injury, Journal of Agricultural and Food Chemistry, 51(6): 1560-1565.

TER BRAAK, C.J.F. 1986. Canonical Correspondence Analysis: A New Eigenvector Technique for Multivariate Direct Gradient Analysis, Ecology, 67(5), pp. 1167-1179. doi: 10.2307/1938672.

TER BRAAK, C.J.F. 1994. Canonical community ordination. Part I: Basic theory and linear methods, Écoscience, 1(2), pp. 127-140. doi: 10.1080/ 11956860. 1994.11682237.

TOMLINSON, P.B. 1966. Anatomical data in the classification of Commelinaceae, Journal of the Linnean Society, Botany, 59(380), pp. 371-395. doi: 10.1111/j.1095-8339.1966.tb00069.x.

WEBSTER, T.M., M.G. BURTON, A.S. CULPEPER, AND A.C. YORK. 2005. Tropical spiderwort (Commelina benghalensis): a tropical invader threatens agroecosystems of the southern United States, Weed technology. BioOne, 19(3), pp. 501-508.

WICKENS, G.E. 1976. The flora of Jebel Morra (Sudan Republic) and its geographical affinities., Kew Bull.Add.Ser.,V.HMSO.London.

WILSON, A.K. 1981. Commelinaceae a review of the distribution, biology and control of the important weeds belonging to this family. Tropical Pest Management, Tropical Pest Management, 27, pp. 405-418.

WORLD HEALTH ORGANIZATION TECHNICAL REPORT SERIES. 1985. Energy and Protein

Requirements. Report of a joint FAO/WHO/UNU Expert Consultation, World Health Organization

YOUNES, H.A., A. EL-SAMIE, S. MOHAMED, AND M. AFIFI. 1977. Comprehensive study on some soils of the River terraces in Ismailia region, Egypt. Desert, Egypt.Desert inst. Bull., 27(2), :107-118.

ZHANG, L., X. XUE, Y. JIE, L. JIN, X. ZHU, AND Z. ZHU. 2016. L-proline: a highly effective cryoprotectant for mouse oocyte vitrification, Scientific Reports. Nature Publishing Group, 6(1), : 26326. 


\section{الوعلان البنغالى النوع الغازي : خطوة نحو الفلورا البيولوجية في مصر هدى على عبد الحميد، منى محمودالبوص2 2.

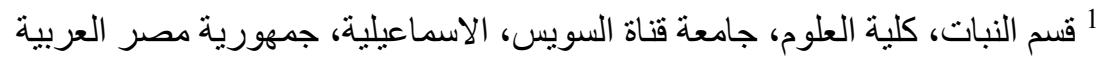

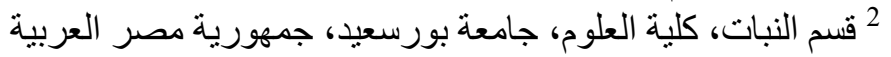 \\ الملخص العربي}

استهدف البحث اجراء دراسة متكاملة لنبات الوعلان البنغالى Commelina benghalensisL من النواحى الظاهرية والتشريحية، كما أجريت دراسات بيئية لوصف التركيب الفلورى لمجتمعه النباتى وتقييم العوامل التي تؤثر على غزوه لمنطقة الدراسة. و علاوة على ذلك تم استقصاءالمكونات الكيميائية و القيمة الغذائية والمعادن ونواتج الأيض الثانوية للنبات ومن خلال جهاز GC / MS (كروماتو غر افيا الغاز - مطياف الكتلة) تم استكثاف طبيعة المركبات النشطة حيويا وتقييم استخداماتها المحتملة. و أسفرت الدراسة البيئية عن تسجيل سبعة و أربعين نوعًا من الحشائش المرتبطة بنبات الوعلان البنغالى، تنتمى إلى 21فصيلة نباتية

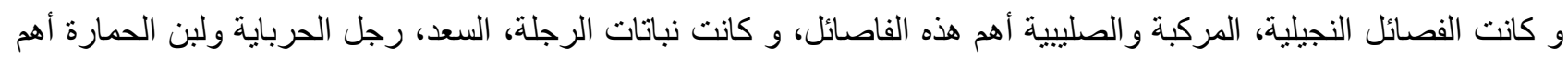
النباتات الدصاحبة له: كما وجد أن طر از الحوليات كان الأكثر شيو عا (78.7٪) بالمنطقة. و أظهر التحليل الفلورى سيادة العناصر الاستوائية القديمة والعالمية والمتوسطية و أشار تحليل برنامج التوزيع التطابقى الكنسي (CCA) إلى أن الأس الهيدروجيني،

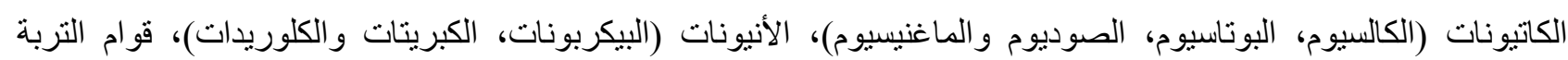

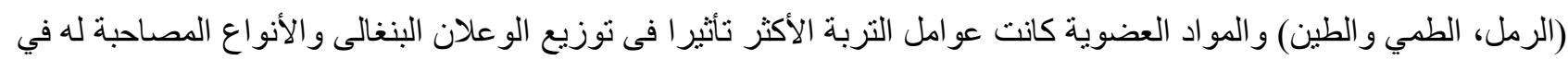
منطقة الدر اسة. كما سجلت الدراسة أعلى قيم للعصارية و متوسط مساحة سطح الورقة لنبات الو علان البنغالى في بساتين المانجو،

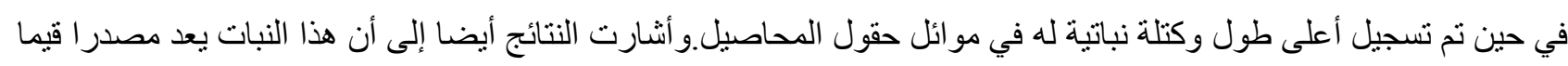

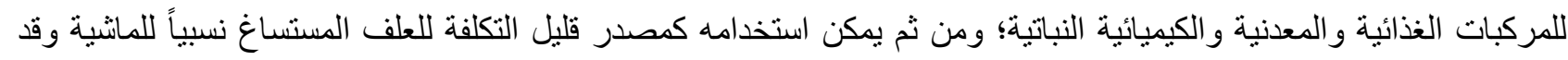

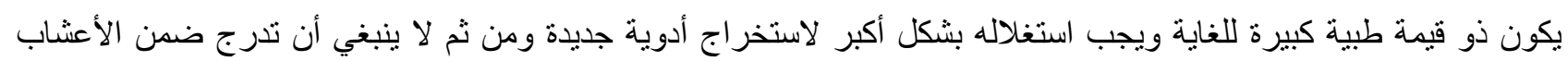
الضارة. كما أنه له القدرة على العيش فى بيئات متعددة ويتحمل الاجهاد البيئى. 